\title{
Variational formulation of plasma dynamics
}

Cite as: Phys. Plasmas 27, 022110 (2020); https://doi.org/10.1063/1.5139315

Submitted: 19 November 2019 . Accepted: 21 January 2020 . Published Online: 13 February 2020

G. O. Ludwig (D)
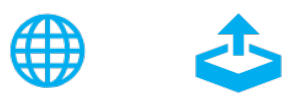

View Online

Export Citation

\section{ARTICLES YOU MAY BE INTERESTED IN}

Quantum hydrodynamics for plasmas-Quo vadis?

Physics of Plasmas 26, 090601 (2019); https://doi.org/10.1063/1.5097885

Perspectives, frontiers, and new horizons for plasma-based space electric propulsion Physics of Plasmas 27, 020601 (2020); https://doi.org/10.1063/1.5109141

Hot electrons between cold walls

Physics of Plasmas 27, 022302 (2020); https://doi.org/10.1063/1.5134499

\section{AVs Quantum Science}




\title{
Variational formulation of plasma dynamics
}

\author{
Cite as: Phys. Plasmas 27, 022110 (2020); doi: 10.1063/1.5139315 \\ Submitted: 19 November 2019 - Accepted: 21 January 2020 . \\ Published Online: 13 February 2020
}
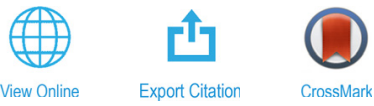

\section{G. O. Ludwig}

\begin{abstract}
AFFILIATIONS
National Institute for Space Research, 12227-010 São José dos Campos, SP, Brazil and National Commission of Nuclear Energy, 22294-900 Rio de Janeiro, RJ, Brazil
\end{abstract}

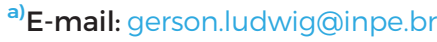

\begin{abstract}
Hamilton's principle is applied to obtain the equations of motion for fully relativistic collision-free plasma. The variational treatment is presented in both the Eulerian and Lagrangian frameworks. A Clebsch representation of the plasma fluid equations shows the connection between the Lagrangian and Eulerian formulations, clarifying the meaning of the multiplier in Lin's constraint. The existence of a fully relativistic hydromagnetic Cauchy invariant is demonstrated. The Lagrangian approach allows a straightforward determination of the Hamiltonian density and energy integral. The definitions of momentum, stress, and energy densities allow one to write the conservation equations in a compact and covariant form. The conservation equations are also written in an integral form with an emphasis on a generalized virial theorem. The treatment of boundary conditions produces a general expression for energy density distribution in plasma fluid.
\end{abstract}

Published under license by AIP Publishing. https://doi.org/10.1063/1.5139315

\section{INTRODUCTION}

Variational formulations of fluid dynamics have a long history, but the modern presentation was mostly developed in the 1950s and 1960s. ${ }^{1-5}$ Although the Lagrangian and Eulerian formulations were firmly established during this period, they continue to be an important topic of research. In the Lagrangian formulation, each fluid element can be treated as a particle, whose position is followed in time. Accordingly, each fluid element is identified by a spatial integration, which is performed over the position occupied by the element at the initial time $t=0$. In the Eulerian approach, the integration region is fixed in space, and the fluid element passes through this position at the instant of time $t$. For the fixed initial position and changing $t$, transformation from Lagrangian to Eulerian variables specifies the trajectory of the fluid element. This continuous transformation (Lagrangian map) of configuration space into itself, parameterized by time $t$, forms the concept of fluid flow.

The canonical Lagrangian picture is akin to particle physics and quantum mechanics, providing a convenient tool in the treatment of symmetries and conserved quantities. ${ }^{6-8}$ On the other hand, the noncanonical Eulerian equations are closed in the macroscopic fluid variables, giving the general picture of flow. These equations can be, in principle, solved without finding the trajectory of all the fluid elements. They are generally preferred in practical applications and in the treatment of boundary conditions. Both formulations are equivalent. ${ }^{9}$

Although not variational, relativistic formulations of the equations of motion for perfect fluids have been presented in many textbooks. ${ }^{10,11}$ Nevertheless, due to its importance in astrophysics, since early efforts, the variational formulation has been extended to cover relativistic perfect fluids. ${ }^{12-15}$ It should also be mentioned that the topic of invariants in fluid flow, first advanced by Cauchy in 1815, continues to be of interest in current research. ${ }^{16-18}$ Invariants appear in varied applications of plasma physics. ${ }^{19-21}$

Plasma fluid equations possess an energy integral and a subsidiary energy principle derived from a linearized Lagrangian form of hydromagnetic equations. ${ }^{22,23}$ This energy principle has been successfully used in the stability analysis of magnetically confined plasmas. However, it seems that a complete variational formulation of fully relativistic plasma fluid equations, including the full set of Maxwell's equations and conserved quantities, is lacking in the literature. The present paper is partly based on the author's unpublished Ph.D. thesis. ${ }^{24}$ It is deemed worthwhile to present these well-established results in a consistent form due both to its inherent importance and in the interest of applying the same formulation to extended theories of gravitoelectromagnetism. Some comments about these applications will be presented in Sec. VIII. Due to its fully relativistic content, the results are applicable in astrophysical plasma problems, although not including gravitation in their present form. This is the quest of extended gravitoelectromagnetism.

Equations of motion of plasma fluid can be derived in a fully covariant form. However, in applications, the equations become more transparent without using the covariant formalism. In particular, the transformation between the Eulerian and Lagrangian frameworks, as 
well as the derivation of a hydromagnetic Cauchy invariant, becomes straightforward using the Jacobian matrix of configuration space in the transformation. This approach, proposed by Cauchy, is fully explored in the present paper. It makes possible to demonstrate the existence, possibly for the first time, of a Cauchy invariant for threedimensional relativistic, compressible, and rotational plasma flows in the presence of a magnetic field. Nevertheless, the covariant form of the dynamical equations is readily obtained after defining the energy and momentum densities and the stress tensor for each component of the plasma fluid. The covariant form of the conservation equations is presented in Appendix A.

The content of this paper is organized as follows: Sec. II develops the variational principle for fluid plasma in both its Eulerian (Subsection II B) and Lagrangian (Subsection IIC) forms. In the sequence, Sec. III presents the fluid equations in the Clebsch parameterization, including a discussion about helicity injection in plasma. Then, Sec. IV demonstrates a hydromagnetic Cauchy invariant. This is followed by a derivation of the Hamiltonian density and the energy integral in Sec. V. The conservation equations are put in compact and integral forms in Sec. VI. Section VII is dedicated to the boundary conditions and the energy density balance. Finally, Sec. VIII presents some comments, notably concerning the extension of electromagnetic theory to gravitoelectromagnetism.

\section{HAMILTON'S PRINCIPLE AND THE PLASMA FLUID EQUATIONS}

Hamilton's principle provides an elegant derivation of the equations of motion of a fluid. ${ }^{1,2,4}$ A brief account of the concepts of classical field theory and notation used in this paper is given in Subsection A.

\section{A. Basic concepts of classical field theory}

In its Eulerian form (fixed frame), Hamilton's principle states that the fluid equations of motion for generalized field coordinates $\varphi(\boldsymbol{r}, t)$ can be derived from a Lagrangian density $\mathcal{L}(\varphi, \dot{\varphi}, \nabla \varphi, t)$ by the variational principle, ${ }^{25}$

$$
\delta \int d^{3} r d t \mathcal{L}=0 .
$$

The virtual displacements are constructed for constant $r$ and $t$ so that Hamilton's principle and integration by parts give

$$
\begin{aligned}
\int d^{3} r d t\left[\frac{\partial \mathcal{L}}{\partial \varphi} \delta \varphi+\frac{\partial \mathcal{L}}{\partial \dot{\varphi}} \delta \dot{\varphi}+\frac{\partial \mathcal{L}}{\partial(\nabla \varphi)} \cdot \delta(\nabla \varphi)\right] \\
=\int d^{3} r d t\left[\frac{\partial \mathcal{L}}{\partial \varphi}-\frac{\partial}{\partial t}\left(\frac{\partial \mathcal{L}}{\partial \dot{\varphi}}\right)-\nabla \cdot\left(\frac{\partial \mathcal{L}}{\partial(\nabla \varphi)}\right)\right] \delta \varphi=0 .
\end{aligned}
$$

For arbitrary variation of the field coordinate $\varphi$, with vanishing variation $\delta \varphi$ at the end points, this leads to the Euler-Lagrange equation,

$$
\frac{\partial \mathcal{L}}{\partial \varphi}=\frac{\partial}{\partial t}\left(\frac{\partial \mathcal{L}}{\partial \dot{\varphi}}\right)+\nabla \cdot\left(\frac{\partial \mathcal{L}}{\partial(\nabla \varphi)}\right)
$$

This equation can be written in the following form:

$$
\frac{\delta \mathcal{L}}{\delta \varphi}-\frac{\partial}{\partial t}\left(\frac{\partial \mathcal{L}}{\partial \dot{\varphi}}\right)=0
$$

where

$$
\frac{\delta \mathcal{L}}{\delta \varphi}=\frac{\partial \mathcal{L}}{\partial \varphi}-\nabla \cdot\left(\frac{\partial \mathcal{L}}{\partial(\nabla \varphi)}\right)
$$

denotes the variational or functional derivative. The momentum density conjugate to $\varphi$ is defined by

$$
\pi(\boldsymbol{r}, t)=\frac{\partial \mathcal{L}}{\partial \dot{\varphi}},
$$

so that the Euler-Lagrange equation implies

$$
\frac{\delta \mathcal{L}}{\delta \varphi}=\dot{\pi}
$$

The Hamiltonian density is defined by

$$
\mathcal{H}\left(\varphi_{\alpha}, \pi_{\alpha}, \nabla \varphi_{\alpha}, t\right)=\sum_{\alpha} \pi_{\alpha} \dot{\varphi}_{\alpha}-\mathcal{L}\left(\varphi_{\alpha}, \dot{\varphi}_{\alpha}, \nabla \varphi_{\alpha}, t\right),
$$

with a sum over field coordinates. Using this definition of $\mathcal{H}$, the variation of the total Hamiltonian becomes

$$
\begin{aligned}
\delta H= & \sum_{\alpha} \int\left[\dot{\varphi}_{\alpha} \delta \pi_{\alpha}+\pi_{\alpha} \delta \dot{\varphi}_{\alpha}-\frac{\partial \mathcal{L}}{\partial \varphi_{\alpha}} \delta \varphi_{\alpha}\right. \\
& \left.-\frac{\partial \mathcal{L}}{\partial \cdot \varphi_{\alpha}} \delta \dot{\varphi}_{\alpha}-\frac{\partial \mathcal{L}}{\partial\left(\nabla \varphi_{\alpha}\right)} \cdot \delta\left(\nabla \varphi_{\alpha}\right)\right] d^{3} r .
\end{aligned}
$$

Substituting the definition of $\pi_{\alpha}$ and the expression of the Euler-Lagrange equation for the field coordinate $\varphi_{\alpha}$ gives

$$
\delta H=\sum_{\alpha} \int\left[\dot{\varphi}_{\alpha} \delta \pi_{\alpha}-\frac{\partial}{\partial t}\left(\frac{\partial \mathcal{L}}{\partial \dot{\varphi}_{\alpha}}\right) \delta \varphi_{\alpha}-\nabla \cdot\left(\frac{\partial \mathcal{L}}{\partial\left(\nabla \varphi_{\alpha}\right)} \delta \varphi_{\alpha}\right)\right] d^{3} r .
$$

Hence,

$$
\delta H=\sum_{\alpha} \int\left(\dot{\varphi}_{\alpha} \delta \pi_{\alpha}-\dot{\pi}_{\alpha} \delta \varphi_{\alpha}\right) d^{3} r-\sum_{\alpha} \oint\left(\frac{\partial \mathcal{L}}{\partial\left(\nabla \varphi_{\alpha}\right)} \delta \varphi_{\alpha}\right) \cdot d^{2} \mathbf{r},
$$

where the surface integral vanishes for a system with vanishing $\delta \varphi$ at the end points (closed system). In this derivation, it was assumed that $\mathcal{H}$ and $\mathcal{L}$ do not depend explicitly on the time. In the Hamiltonian formulation, $\mathcal{H}$ is a function of $\pi_{\alpha}, \varphi_{\alpha}, \nabla \varphi_{\alpha}$ so that

$$
\begin{aligned}
\delta H= & \sum_{\alpha} \delta \int \mathcal{H}\left(\pi_{\alpha}, \varphi_{\alpha}, \nabla \varphi_{\alpha}\right) d^{3} r \\
= & \sum_{\alpha} \int\left(\frac{\partial \mathcal{H}}{\partial \pi_{\alpha}} \delta \pi_{\alpha}+\frac{\partial \mathcal{H}}{\partial \varphi_{\alpha}} \delta \varphi_{\alpha}+\frac{\partial \mathcal{H}}{\partial\left(\nabla \varphi_{\alpha}\right)} \cdot \delta\left(\nabla \varphi_{\alpha}\right)\right) d^{3} r \\
= & \sum_{\alpha} \int\left[\frac{\partial \mathcal{H}}{\partial \pi_{\alpha}} \delta \pi_{\alpha}+\frac{\partial \mathcal{H}}{\partial \varphi_{\alpha}} \delta \varphi_{\alpha}-\nabla \cdot\left(\frac{\partial \mathcal{H}}{\partial\left(\nabla \varphi_{\alpha}\right)}\right) \delta \varphi_{\alpha}\right] d^{3} r \\
& +\sum_{\alpha} \oint\left(\frac{\partial \mathcal{H}}{\partial\left(\nabla \varphi_{\alpha}\right)} \delta \varphi_{\alpha}\right) \cdot d^{2} \mathbf{r} .
\end{aligned}
$$

Comparing the two expressions of $\delta H$ leads to the Hamiltonian form of the field dynamic equations 


$$
\left\{\begin{array}{l}
\dot{\varphi}_{\alpha}=\frac{\partial \mathcal{H}}{\partial \pi_{\alpha}}=\frac{\delta \mathcal{H}}{\delta \pi_{\alpha}}, \\
\dot{\pi}_{\alpha}=-\frac{\partial \mathcal{H}}{\partial \varphi_{\alpha}}+\nabla \cdot\left(\frac{\partial \mathcal{H}}{\partial\left(\nabla \varphi_{\alpha}\right)}\right)=-\frac{\delta \mathcal{H}}{\delta \varphi_{\alpha}} .
\end{array}\right.
$$

Here, the functional derivative notation indicates the variation of $\mathcal{H}$ for small differences $\delta \varphi_{\alpha}$ in the path followed by the field variable $\varphi_{\alpha}$,

$$
\frac{\delta \mathcal{H}}{\delta \varphi_{\alpha}}=\frac{\partial \mathcal{H}}{\partial \varphi_{\alpha}}-\nabla \cdot\left(\frac{\partial \mathcal{H}}{\partial\left(\nabla \varphi_{\alpha}\right)}\right) .
$$

If $F$ is the volume integral of a density function $\mathcal{F}\left(\pi_{\alpha}, \varphi_{\alpha}, \nabla \varphi_{\alpha}, t\right)$, its time derivative is given by

$$
\begin{aligned}
\frac{d F}{d t} & =\frac{d}{d t} \int \mathcal{F} d^{3} r \frac{\partial F}{\partial t}+\sum_{\alpha} \int\left(\frac{\delta \mathcal{F}}{\delta \varphi_{\alpha}} \dot{\varphi}_{\alpha}+\frac{\delta \mathcal{F}}{\delta \pi_{\alpha}} \dot{\pi}_{\alpha}\right) d^{3} r \\
& =\frac{\partial F}{\partial t}+\sum_{\alpha} \int\left(\frac{\delta \mathcal{F}}{\delta \varphi_{\alpha}} \frac{\delta \mathcal{H}}{\delta \pi_{\alpha}}-\frac{\delta \mathcal{F}}{\delta \pi_{\alpha}} \frac{\delta \mathcal{H}}{\delta \varphi_{\alpha}}\right) d^{3} r .
\end{aligned}
$$

Hence,

$$
\frac{d F}{d t}=\frac{\partial F}{\partial t}+\sum_{\alpha} \int\{\mathcal{F}, \mathcal{H}\} d^{3} r,
$$

where $\{\mathcal{F}, \mathcal{H}\}$ is the classical Poisson bracket for $\mathcal{F}$ and $\mathcal{H}$. This relation constitutes the noncanonical Hamiltonian representation of a system. In particular, taking $\mathcal{F}=\mathcal{H}$ leads to the energy integral

$$
\frac{d H}{d t}=\frac{d}{d t} \int \mathcal{H} d^{3} r=0 .
$$

\section{B. Eulerian variational principle}

The Lagrangian density for a multi-species relativistic perfect plasma is taken in the following form:

$$
\mathcal{L}=\sum_{q}(-U-\rho \phi+\mathbf{j} \cdot \mathbf{A})+\frac{1}{2}\left(\epsilon_{0} E^{2}-\frac{B^{2}}{\mu_{0}}\right),
$$

where the summation extends over all the particle species designated by the charge $q$. For simplicity, no indices will be used to distinguish the field variables of the various species. Each species evolves independently with a flow velocity $\boldsymbol{v}$ (collision-free plasma) except for the Vlasov mean-field interactions. Nevertheless, a small level of randomizing must be assumed validating the hydrodynamics concepts and leading to a scalar pressure. The plasma energy density $U$ is given by the sum of the mass and thermal energy densities,

$$
U=\frac{n m c^{2}}{\gamma}+\frac{p}{\gamma_{A}-1}
$$

The Lorentz factor is

$$
\gamma=\frac{1}{\sqrt{1-\beta^{2}}}=\frac{1}{\sqrt{1-v^{2} / c^{2}}},
$$

the fluid pressure for a perfect gas is $p=n k_{B} T$, and the "adiabatic" coefficient $\gamma_{A}$ for a relativistic plasma is a function of the temperature $T$ (cf. Appendix A). The interaction energy density between the charged particles and the electromagnetic field is $-\rho \phi+\boldsymbol{j} \cdot \boldsymbol{A}$, where $\rho=n q$ is the charge density of the plasma species $q$ and $\boldsymbol{j}=\rho \boldsymbol{v}$ is the current density. The electromagnetic field variables $\boldsymbol{E}$ and $\boldsymbol{B}$ are related to the potentials $\phi$ and $A$ by

$$
\begin{aligned}
& \boldsymbol{E}=-\boldsymbol{\nabla} \phi-\frac{\partial \boldsymbol{A}}{\partial t}, \\
& \boldsymbol{B}=\boldsymbol{\nabla} \times \boldsymbol{A} .
\end{aligned}
$$

These relations lead to Faraday's law

$$
\boldsymbol{\nabla} \times \boldsymbol{E}=-\frac{\partial \boldsymbol{B}}{\partial t}
$$

and to the magnetic Gauss's law

$$
\nabla \cdot B=0 .
$$

The free-field Lagrangian can be written in terms of the first electromagnetic field invariant $\Lambda$,

$$
\frac{1}{2}\left(\epsilon_{0} E^{2}-\frac{B^{2}}{\mu_{0}}\right)=-\frac{\Lambda}{4 \mu_{0}} .
$$

The second electromagnetic field invariant is

$$
M=-\frac{4(\boldsymbol{E} \cdot \boldsymbol{B})}{c} .
$$

This second invariant is used in the strong magnetic field assumption, which requires that the electric and magnetic fields are approximately perpendicular, i.e., $M \cong 0$, leading to a non-scalar pressure tensor and to the double adiabatic CGL equations of state for the plasma. ${ }^{26,27}$ Since anisotropic plasma states are not considered in the present paper, constraints involving $M$ are not imposed on the plasma fluid dynamics.

The hydroelectromagnetic equations of motion are obtained from the expression of the Lagrangian density by the variational procedure described in Subsection II A The field coordinates are

$$
\begin{array}{ll}
\phi, A & - \text { electromagnetic field potentials, } \\
\boldsymbol{v} & - \text { fluid velocity, } \\
n & - \text { fluid number density, } \\
s & - \text { proper entropy of a fluid element, } \\
r_{0} & - \text { Lagrangian coordinate of a particle. }
\end{array}
$$

The vector field $\boldsymbol{r}_{0}(\boldsymbol{r}, t)$ establishes the initial position of the fluid element (particle) that occupies the position $r$ at time $t$. The above field variables are not completely independent; they must satisfy the following constraints:

$$
\begin{array}{ll}
\dot{n}+\boldsymbol{\nabla} \cdot(n \boldsymbol{v})=0 & - \text { continuity } \\
d s / d t=0 & - \text { isentropic flow, } \\
d \boldsymbol{r}_{0} / d t=0 & - \text { identity of particles. }
\end{array}
$$

Here, $\dot{n}=\partial n / \partial t$ indicates the partial time derivative. It gives the rate of change of the density $n$ at a stationary point $r$. The total or convective time derivative $d / d t \equiv \partial / \partial t+\boldsymbol{v} \cdot \nabla$ gives the rate of change of a quantity moving instantaneously with the velocity $\boldsymbol{v}$. It describes the advection by fluid motion. As for the fluid constraints: (1) Fluid continuity is a kinematic condition of fluid motion (cf. Appendix B). This condition corresponds to the conservation of the number of particles. 
(2) The specific entropy $s$ is constant in time at any point that moves along with the perfect fluid without irreversible processes taking place (cf. Appendix A). (3) The last condition above implies that there is a one-to-one correspondence between Lagrangian and Eulerian coordinates (cf. Appendix B). This constraint was introduced by Lin so that the total set of constraints gives rise to a Herivel-Lin flow. ${ }^{2}$ The meaning of the Lagrangian multiplier in Lin's constraint is clarified in Sec. III. Hence, Hamilton's principle can be put in the following form:

$$
\delta \int d^{3} r d t\left[\mathcal{L}+a(\dot{n}+\nabla \cdot(n \boldsymbol{v}))+b n \frac{d s}{d t}+n \boldsymbol{c} \cdot\left(\frac{d \boldsymbol{r}_{0}}{d t}\right)\right]=0,
$$

where $a, b$, and $c$ constitute a set of Lagrangian multipliers.

Now, $\phi, \boldsymbol{A}, \boldsymbol{v}, n, s$, and $\boldsymbol{r}_{0}$ are independent functions of $\boldsymbol{r}$ and $t$ that can be varied. In carrying out the variation, the volume of integration is kept fixed, in the Eulerian sense. Furthermore, the thermodynamic potentials satisfy the fundamental law (cf. Appendix A),

$$
\frac{1}{k_{B}} d s=\frac{1}{k_{B} T} d\left(\frac{k_{B} T}{\gamma_{A}-1}\right)+\frac{\gamma_{A}}{\gamma_{A}-1} \frac{d \gamma}{\gamma}-\frac{d n}{n} .
$$

An equation of state, which is a subsidiary condition to the fluid flow, must also be given. For a perfect fluid, this is simply the perfect gas law $p=n k_{B} T$. Hence, an Euler-Lagrange equation for each field coordinate $\varphi$ can be derived from the modified Lagrangian function $\mathcal{L}^{\prime}$ given by

$$
\begin{aligned}
\mathcal{L}^{\prime}= & \sum_{q}\left[-\frac{n m c^{2}}{\gamma}-\frac{n k_{B} T}{\gamma_{A}-1}-n q \phi+n q \boldsymbol{v} \cdot \mathbf{A}\right. \\
& +a(\dot{n}+\boldsymbol{v} \cdot \boldsymbol{\nabla} n+n \boldsymbol{\nabla} \cdot \boldsymbol{v})+b n(\dot{\boldsymbol{s}}+\boldsymbol{v} \cdot \boldsymbol{\nabla} s) \\
& \left.+n \boldsymbol{c} \cdot\left(\dot{\boldsymbol{r}_{0}}+\boldsymbol{v} \cdot \nabla \boldsymbol{r}_{0}\right)\right]+\frac{1}{2}\left(\epsilon_{0} E^{2}-\frac{B^{2}}{\mu_{0}}\right) .
\end{aligned}
$$

The Euler-Lagrange equations are obtained by straightforward differentiation and listed in the following:

$$
\begin{aligned}
& \text { (i) } \delta \phi \quad \nabla \cdot \boldsymbol{E}=\frac{1}{\epsilon_{0}} \sum_{q} \rho, \\
& \text { (ii) } \delta \boldsymbol{A} \quad \boldsymbol{\nabla} \times \boldsymbol{B}=\mu_{0} \sum_{q} j+\frac{1}{c^{2}} \frac{\partial \boldsymbol{E}}{\partial t} \text {, } \\
& \text { (iii) } \delta \boldsymbol{v} \quad \gamma m \boldsymbol{v}-\gamma^{3} \frac{\partial}{\partial \gamma}\left(\frac{k_{B} T}{\gamma_{A}-1}\right)_{n, s} \frac{\boldsymbol{v}}{c^{2}}+q \boldsymbol{A}-\nabla a \\
& +b \boldsymbol{\nabla} s+\left(\boldsymbol{\nabla} \mathbf{r}_{0}\right) \cdot c=0, \\
& \text { (iv) } \delta n \frac{d a}{d t}=-\frac{m c^{2}}{\gamma}-\frac{k_{B} T}{\gamma_{A}-1}-n \frac{\partial}{\partial n}\left(\frac{k_{B} T}{\gamma_{A}-1}\right)_{\gamma, s} \\
& -q \phi+q \boldsymbol{v} \cdot \boldsymbol{A}, \\
& \text { (v) } \delta s \quad \frac{d b}{d t}=-\frac{\partial}{\partial s}\left(\frac{k_{B} T}{\gamma_{A}-1}\right)_{n, \gamma}, \\
& \text { (vi) } \delta r_{0} \quad \frac{d c}{d t}=0 .
\end{aligned}
$$

Equations (i) and (ii) are the laws of Gauss and Ampère, respectively. Equation (iii) gives the fluid velocity $v$ in terms of the vector potential $A$, the scalar potentials $a$ and $s$, and the deformations (strains and rotations) in the initial fluid positions $\boldsymbol{r}_{0}$. Defining a relativistic inertia factor $\alpha$ by

$$
\alpha=\gamma\left[1-\frac{\gamma^{2}}{m c^{2}} \frac{\partial}{\partial \gamma}\left(\frac{k_{B} T}{\gamma_{A}-1}\right)_{n, s}\right],
$$

the Euler-Lagrange equation (iii) can be written in the compact form,

$$
m \alpha \boldsymbol{v}+q \boldsymbol{A}=\boldsymbol{\nabla} a-b \boldsymbol{\nabla} \boldsymbol{s}-\boldsymbol{c} \cdot\left(\boldsymbol{\nabla} \boldsymbol{r}_{0}\right)^{T},
$$

where the superscript $T$ denotes the transposed dyadic. Note that this equation has the form of a Clebsch representation for the canonical momentum $m \alpha \boldsymbol{v}+q \boldsymbol{A}$ of a fluid element. The Euler-Lagrange equations (iv), (v), and (vi) are equations of motion for the Lagrange multipliers $a, b$, and $c$. These equations can be used to eliminate the Lagrange multipliers from equation (iii). This can be accomplished by means of the following relations, which can be obtained using vector and dyadic relations, the constraints $d s / d t=d r_{0} / d t=0$, the Euler-Lagrange equation $d \boldsymbol{c} / d t=0$, and the identity $\boldsymbol{\nabla} \times \boldsymbol{r}_{0}=0$ :

$$
\begin{aligned}
\frac{d}{d t}(\nabla a) & =\nabla \frac{d a}{d t}-\nabla \boldsymbol{v} \cdot(\nabla a), \\
\frac{d}{d t}(b \nabla s) & =(\nabla s) \frac{d b}{d t}-\nabla \boldsymbol{v} \cdot(b \nabla s), \\
\frac{d}{d t}\left[\left(\boldsymbol{\nabla} \boldsymbol{r}_{0}\right) \cdot \boldsymbol{c}\right] & =-(\boldsymbol{\nabla} \boldsymbol{v}) \cdot\left(\boldsymbol{\nabla} \boldsymbol{r}_{0}\right) \cdot \boldsymbol{c} .
\end{aligned}
$$

The total time derivative of Euler-Lagrange's equation (iii) in the form (33) gives

$$
\begin{aligned}
\frac{d}{d t}(m \alpha \boldsymbol{v}+q \boldsymbol{A})= & \frac{d}{d t}\left[\nabla a-b \nabla s-\left(\nabla \boldsymbol{r}_{0}\right) \cdot \boldsymbol{c}\right] \\
= & \nabla \frac{d a}{d t}-\nabla \boldsymbol{v} \cdot(\boldsymbol{\nabla} a)-(\boldsymbol{\nabla} s) \frac{d b}{d t} \\
& +\nabla \boldsymbol{v} \cdot(b \nabla s)+(\nabla v) \cdot\left(\boldsymbol{\nabla} \boldsymbol{r}_{0}\right) \cdot \boldsymbol{c}
\end{aligned}
$$

and the scalar pre-multiplication by $\nabla \boldsymbol{v}$ gives

$$
\boldsymbol{\nabla} \boldsymbol{v} \cdot(m \alpha \boldsymbol{v}+q \boldsymbol{A})=\boldsymbol{\nabla} \boldsymbol{v} \cdot\left[\boldsymbol{\nabla} a-b \boldsymbol{\nabla}-\left(\boldsymbol{\nabla} \boldsymbol{r}_{0}\right) \cdot c\right] .
$$

The addition of these two equations yields

$$
\frac{d}{d t}(m \alpha \boldsymbol{v}+q \boldsymbol{A})+\boldsymbol{\nabla} \boldsymbol{v} \cdot(m \alpha \boldsymbol{v}+q \boldsymbol{A})=\boldsymbol{\nabla} \frac{d a}{d t}-(\nabla \boldsymbol{s}) \frac{d b}{d t} .
$$

Finally, using equations (iv) and (v), the remaining Lagrange multipliers $a$ and $b$ are eliminated from the equation of momentum conservation,

$$
\begin{aligned}
\frac{d}{d t}(m \alpha \boldsymbol{v}+q \boldsymbol{A})+\boldsymbol{\nabla} \boldsymbol{v} \cdot(m \alpha \boldsymbol{v}+q \boldsymbol{A}) \\
=-q \boldsymbol{\nabla} \phi+q \boldsymbol{\nabla}(\boldsymbol{v} \cdot \boldsymbol{A})-c^{2} \boldsymbol{\nabla} \gamma^{-1}-\boldsymbol{\nabla}\left(\frac{k_{B} T / m}{\gamma_{A}-1}\right) \\
\quad-\boldsymbol{\nabla}\left[n \frac{\partial}{\partial n}\left(\frac{k_{B} T / m}{\gamma_{A}-1}\right)_{\gamma, s}\right]+(\boldsymbol{\nabla} s) \frac{\partial}{\partial s}\left(\frac{k_{B} T / m}{\gamma_{A}-1}\right)_{n, \gamma} .
\end{aligned}
$$

Maxwell's differential relations can be determined from the fundamental law of thermodynamics,

$$
\frac{1}{k_{B}} d s=\frac{1}{k_{B} T} d\left(\frac{k_{B} T}{\gamma_{A}-1}\right)+\frac{\gamma_{A}}{\gamma_{A}-1} \frac{d \gamma}{\gamma}-\frac{d n}{n} .
$$


Thus,

$$
\left\{\begin{array}{l}
\frac{\partial}{\partial n}\left(\frac{k_{B} T}{\gamma_{A}-1}\right)_{\gamma, s}=\frac{k_{B} T}{n}, \\
\frac{\partial}{\partial \gamma}\left(\frac{k_{B} T}{\gamma_{A}-1}\right)_{n, s}=-\frac{\gamma_{A}}{\gamma_{A}-1} \frac{k_{B} T}{\gamma}, \\
\frac{\partial}{\partial s}\left(\frac{k_{B} T}{\gamma_{A}-1}\right)_{n, \gamma}=T .
\end{array}\right.
$$

These relations reduce the equation of momentum conservation to the following form:

$$
\begin{aligned}
\frac{d}{d t}(m \alpha \boldsymbol{v}+q \boldsymbol{A})+\boldsymbol{\nabla} \boldsymbol{v} \cdot(m \alpha \boldsymbol{v}+q \boldsymbol{A}) \\
=-q \boldsymbol{\nabla} \phi+q \boldsymbol{\nabla}(\boldsymbol{v} \cdot \boldsymbol{A})-m c^{2} \boldsymbol{\nabla} \gamma^{-1} \\
\quad-\boldsymbol{\nabla}\left(k_{B} T\right)+k_{B} T\left(\frac{\gamma_{A}}{\gamma_{A}-1} \frac{\nabla \gamma}{\gamma}-\frac{\nabla n}{n}\right),
\end{aligned}
$$

where

$$
\alpha=\gamma\left(1+\frac{\gamma_{A}}{\gamma_{A}-1} \frac{\gamma k_{B} T}{m c^{2}}\right) .
$$

Note that the thermal motion increases the fluid inertia. These relativistic effects may strongly affect the reconnection process in astrophysical and inertially confined plasmas. ${ }^{14,28}$ Next, using

$$
\nabla \gamma^{-1}=-\gamma \frac{\nabla v^{2}}{2 c^{2}} \quad \text { and } \quad \nabla \gamma=\gamma^{3} \frac{\nabla v^{2}}{2 c^{2}}
$$

the momentum conservation equation becomes

$$
\begin{aligned}
\frac{d}{d t}(m \alpha \boldsymbol{v})= & -\frac{\boldsymbol{\nabla}\left(n k_{B} T\right)}{n}-q \boldsymbol{\nabla} \phi-q \frac{\partial \boldsymbol{A}}{\partial t} \\
& -q \boldsymbol{v} \cdot \boldsymbol{\nabla} \boldsymbol{A}-q(\boldsymbol{\nabla} \boldsymbol{v}) \cdot \boldsymbol{A}+q \boldsymbol{\nabla}(\boldsymbol{v} \cdot \boldsymbol{A}) .
\end{aligned}
$$

Now, the vector and dyadic relations,

$$
\begin{aligned}
& (\boldsymbol{v} \cdot \boldsymbol{\nabla}) \boldsymbol{A}=(\boldsymbol{\nabla} \boldsymbol{A}) \cdot \boldsymbol{v}-\boldsymbol{v} \times(\boldsymbol{\nabla} \times \boldsymbol{A}), \\
& \boldsymbol{\nabla}(\boldsymbol{v} \cdot \boldsymbol{A})=(\boldsymbol{\nabla} \boldsymbol{v}) \cdot \boldsymbol{A}+(\boldsymbol{\nabla} \boldsymbol{A}) \cdot \boldsymbol{v},
\end{aligned}
$$

give

$$
\frac{d}{d t}(m \alpha \boldsymbol{v})=-\frac{\boldsymbol{\nabla}\left(n k_{B} T\right)}{n}-q\left(\boldsymbol{\nabla} \phi+\frac{\partial \boldsymbol{A}}{\partial t}\right)+q \boldsymbol{v} \times(\boldsymbol{\nabla} \times \boldsymbol{A}) .
$$

Introducing the electromagnetic field components $\boldsymbol{E}=-\boldsymbol{\nabla} \phi-\partial \boldsymbol{A} / \partial t$ and $\boldsymbol{B}=\boldsymbol{\nabla} \times \boldsymbol{A}$, the equation of momentum conservation becomes

$$
\frac{d}{d t}(m \alpha \boldsymbol{v})=-\frac{\nabla p}{n}+q(\boldsymbol{E}+\boldsymbol{v} \times \boldsymbol{B}),
$$

which has the form of a Lorentz force acting on the fluid element.

Multiplying by $n$ and applying the continuity condition $\partial n / \partial t=-\boldsymbol{\nabla} \cdot(n \boldsymbol{v})$, the equation of momentum conservation yields

$$
\begin{gathered}
\frac{\partial}{\partial t}(n m \alpha \boldsymbol{v})+\underbrace{m \alpha \boldsymbol{v} \boldsymbol{\nabla} \cdot(n \boldsymbol{v})+n m \boldsymbol{v} \cdot \boldsymbol{\nabla}(\alpha \boldsymbol{v})}_{\boldsymbol{\nabla} \cdot(n m \alpha \boldsymbol{v} \boldsymbol{v})} \\
=-\boldsymbol{\nabla} p+n q(\boldsymbol{E}+\boldsymbol{v} \times \boldsymbol{B}) .
\end{gathered}
$$

Therefore, the full equation of momentum conservation for each species in a relativistic perfect plasma fluid submitted to the action of an (self-consistent or external) electromagnetic field can be written as

$$
\begin{aligned}
& \frac{\partial}{\partial t}\left[\gamma\left(1+\frac{\gamma_{A}}{\gamma_{A}-1} \frac{\gamma p}{n m c^{2}}\right) n m \boldsymbol{v}\right] \\
& \quad+\boldsymbol{\nabla} \cdot\left[\gamma\left(1+\frac{\gamma_{A}}{\gamma_{A}-1} \frac{\gamma p}{n m c^{2}}\right) n m \boldsymbol{v} \boldsymbol{v}+p \overline{\overline{\boldsymbol{I}}}\right]=\rho \boldsymbol{E}+\boldsymbol{j} \times \boldsymbol{B}
\end{aligned}
$$

where $\overline{\overline{\boldsymbol{I}}}$ is the unit dyadic.

The entropy conservation law $d s=0$ leads to the differential equations of state (cf. Appendix A),

$$
\begin{aligned}
\frac{d p}{p} & =\frac{1}{\gamma k_{B} T} d\left(\frac{\gamma_{A}}{\gamma_{A}-1} \gamma k_{B} T\right) \\
& =\frac{\gamma_{A}^{2}}{\gamma_{A}-1} \frac{\gamma}{n} d\left(\frac{\gamma_{A}-1}{\gamma_{A}} \frac{n}{\gamma}\right) .
\end{aligned}
$$

Thus,

$$
\frac{\nabla p}{n m}=\frac{1}{\gamma} \nabla\left(\frac{\gamma_{A}}{\gamma_{A}-1} \frac{\gamma k_{B} T}{m}\right)
$$

can be used to express the fluid pressure in terms of the temperature,

$$
\begin{aligned}
\frac{d}{d t} & {\left[\gamma\left(1+\frac{\gamma_{A}}{\gamma_{A}-1} \frac{\gamma k_{B} T}{m c^{2}}\right) \boldsymbol{v}\right] } \\
& =-\frac{1}{\gamma} \nabla\left(\frac{\gamma_{A}}{\gamma_{A}-1} \frac{\gamma k_{B} T}{m}\right)+\frac{q}{m}(\boldsymbol{E}+\boldsymbol{v} \times \boldsymbol{B}) .
\end{aligned}
$$

The acceleration in this equation can be written in a compact form in terms of the function $\alpha$,

$$
\frac{d}{d t}(\alpha \boldsymbol{v})=-\frac{c^{2}}{\gamma} \nabla\left(\frac{\alpha}{\gamma}\right)+\frac{q}{m}(\boldsymbol{E}+\boldsymbol{v} \times \boldsymbol{B}) .
$$

Scalar multiplication by $\boldsymbol{v}$ gives

$$
\boldsymbol{v} \cdot \frac{d}{d t}(\alpha \boldsymbol{v})=-\frac{c^{2}}{\gamma} \boldsymbol{v} \cdot \nabla\left(\frac{\alpha}{\gamma}\right)+\frac{\boldsymbol{j} \cdot \boldsymbol{E}}{n m} .
$$

Thus,

$$
\frac{\alpha}{2} \frac{d v^{2}}{d t}+v^{2} \frac{d \alpha}{d t}=-\frac{c^{2}}{\gamma} \frac{d}{d t}\left(\frac{\alpha}{\gamma}\right)+\frac{c^{2}}{\gamma} \frac{\partial}{\partial t}\left(\frac{\alpha}{\gamma}\right)+\frac{\boldsymbol{j} \cdot \boldsymbol{E}}{n m} .
$$

In terms of the Lorentz factor $\gamma$,

$$
\frac{v^{2}}{c^{2}}=1-\frac{1}{\gamma^{2}}, \quad \frac{d v^{2}}{c^{2}}=\frac{2}{\gamma^{3}} d \gamma,
$$

and

$$
\frac{d}{d t}\left(\alpha c^{2}\right)=\frac{1}{\gamma} \frac{\partial}{\partial t}\left(\frac{\alpha c^{2}}{\gamma}\right)+\frac{\boldsymbol{j} \cdot \boldsymbol{E}}{n m} .
$$

Multiplication by the mass density $n m$ gives

$$
\frac{d}{d t}\left(n m c^{2} \alpha\right)-m c^{2} \alpha \frac{d n}{d t}=\frac{n m c^{2}}{\gamma} \frac{\partial}{\partial t}\left(\frac{\alpha}{\gamma}\right)+\boldsymbol{j} \cdot \boldsymbol{E} .
$$

Using the continuity condition, 


$$
\frac{1}{n} \frac{d n}{d t}=-\nabla \cdot \boldsymbol{v}
$$

it follows that

$$
\frac{\partial}{\partial t}\left(n m c^{2} \alpha\right)+\boldsymbol{\nabla} \cdot\left(n m c^{2} \alpha \boldsymbol{v}\right)=\frac{n m c^{2}}{\gamma} \frac{\partial}{\partial t}\left(\frac{\alpha}{\gamma}\right)+\boldsymbol{j} \cdot \boldsymbol{E} .
$$

Since

$$
d\left(\frac{\alpha}{\gamma}\right)=d\left(\frac{\gamma_{A}}{\gamma_{A}-1} \frac{\gamma k_{B} T}{m c^{2}}\right)=\frac{\gamma d p}{n m c^{2}},
$$

the energy conservation equation becomes

$$
\frac{\partial}{\partial t}\left(n m c^{2} \alpha\right)+\nabla \cdot\left(n m c^{2} \alpha \boldsymbol{v}\right)=\frac{\partial p}{\partial t}+\boldsymbol{j} \cdot \boldsymbol{E} .
$$

With some rearrangement, the energy conservation equation can be written as

$$
\begin{aligned}
& \frac{\partial}{\partial t}\left[\gamma n m c^{2}+\left(\frac{1}{\gamma_{A}-1}+\beta^{2}\right) \gamma^{2} p\right] \\
& \quad+\boldsymbol{\nabla} \cdot\left[\left(\gamma n m c^{2}+\frac{\gamma_{A}}{\gamma_{A}-1} \gamma^{2} p\right) \boldsymbol{v}\right]=\boldsymbol{j} \cdot \boldsymbol{E} .
\end{aligned}
$$

Summary: The variational procedure shows that the momentum conservation equation,

$$
\frac{d}{d t}\left[\gamma\left(1+\frac{\gamma_{A}}{\gamma_{A}-1} \frac{\gamma k_{B} T}{m c^{2}}\right) m \boldsymbol{v}\right]=-\frac{\nabla p}{n}+q(\boldsymbol{E}+\boldsymbol{v} \times \boldsymbol{B}),
$$

describes the plasma fluid flow together with the equation of continuity,

$$
\frac{\partial n}{\partial t}+\nabla \cdot(n \boldsymbol{v})=0,
$$

and Maxwell's equations,

$$
\begin{aligned}
\nabla \cdot \boldsymbol{E} & =\frac{1}{\epsilon_{0}} \sum_{q} \rho \quad \text { Gauss's law }, \\
\boldsymbol{\nabla} \times \boldsymbol{B} & =\mu_{0} \sum_{q} \mathbf{j}+\frac{1}{c^{2}} \frac{\partial \boldsymbol{E}}{\partial t} \quad \text { Ampere's law, }
\end{aligned}
$$

where $\rho$ and $\boldsymbol{j}$ are the charge and current densities corresponding to species $q$

$$
\rho=n q, \quad \boldsymbol{j}=n q \boldsymbol{v} .
$$

The electromagnetic field variables are related to the potentials by

$$
\begin{aligned}
& \boldsymbol{E}=-\nabla \phi-\frac{\partial \boldsymbol{A}}{\partial t}, \\
& \boldsymbol{B}=\boldsymbol{\nabla} \times \boldsymbol{A},
\end{aligned}
$$

which leads to the consistency relations

$$
\begin{aligned}
\boldsymbol{\nabla} \times \boldsymbol{E} & =-\frac{\partial \boldsymbol{B}}{\partial t} \quad \text { Faraday's law } \\
\boldsymbol{\nabla} \cdot \boldsymbol{B} & =0 \quad \text { magnetic Gauss's law }
\end{aligned}
$$

The pressure $p$ is related to the temperature $T$ according to the isentropic flow condition $d s / d t=0$. This is equivalent to the energy conservation equation. The equation of state for a perfect fluid is $p=n k_{B} T$, which can be used to relate the density $n$ to the temperature $T$.

Introducing the weakly relativistic approximation,

$$
\gamma \sim 1+\frac{v^{2}}{2 c^{2}}
$$

the energy conservation equation reduces to

$$
\begin{aligned}
& \frac{\partial}{\partial t}(\underbrace{n m c^{2}}_{\text {dominant }}+n m \frac{v^{2}}{2}+\frac{p}{\gamma_{A}-1}) \\
& \quad+\boldsymbol{\nabla} \cdot(\underbrace{n m c^{2} \boldsymbol{v}}_{\text {dominant }}+n m \frac{v^{2}}{2} \boldsymbol{v}+\frac{\gamma_{A}}{\gamma_{A}-1} p \boldsymbol{v}) \cong \boldsymbol{j} \cdot \boldsymbol{E},
\end{aligned}
$$

where the dominant terms (in the expansion in powers of $1 / c$ ) cancel due to fluid continuity. Similarly, the equation of momentum conservation for charged species reduces to

$$
\frac{\partial}{\partial t}(n m \boldsymbol{v})+\boldsymbol{\nabla} \cdot(n m \boldsymbol{v} \boldsymbol{v}+p \overline{\overline{\boldsymbol{I}}}) \cong \rho \boldsymbol{E}+\boldsymbol{j} \times \boldsymbol{B},
$$

or, in the equivalent Lorentz force form,

$$
m \frac{d \boldsymbol{v}}{d t} \cong-\nabla\left(\frac{\gamma_{A} k_{B} T}{\gamma_{A}-1}\right)+q(\boldsymbol{E}+\boldsymbol{v} \times \boldsymbol{B}) .
$$

Consequently, Hamilton's principle applied to the Lagrangian density $\mathcal{L}$ leads to the required conservation of momentum equation and Maxwell's equations for each species of a collision-free relativistic plasma under the action of a self-consistent electromagnetic field. In Subsection $\mathrm{C}$, the variation of $\mathcal{L}$ will be carried out in Lagrangian coordinates.

\section{Lagrangian variational principle}

In this subsection, the equations of fluid motion are considered within the Lagrangian approach. The variation is applied to the positions of the fluid elements. Changing from Eulerian $(\boldsymbol{r}, t)$ to Lagrangian $\left(\boldsymbol{r}_{0}, \tau\right)$ coordinates, Hamilton's principle becomes

$$
\begin{aligned}
& \delta \int d^{3} r_{0} d \tau J\left[\sum_{q}\left(-\frac{n m c^{2}}{\gamma}-\frac{n k_{B} T}{\gamma_{A}-1}-n q \phi+n q \mathbf{A} \cdot \frac{\partial \xi}{\partial \tau}\right)\right. \\
& \left.\quad+\frac{1}{2}\left(\epsilon_{0} E^{2}-\frac{B^{2}}{\mu_{0}}\right)\right]=0,
\end{aligned}
$$

$\overline{\bar{J}}$ is the Jacobian dyadic,

$$
\overline{\bar{J}}=\nabla_{0} r=\nabla_{0}\left(r_{0}+\xi\right)=\overline{\bar{I}}+\nabla_{0} \xi,
$$

and $J=|\overline{\overline{\boldsymbol{J}}}|$ its determinant. In this variational principle, the integration extends over the fixed initial positions, and the field variables are considered as functions of $\boldsymbol{r}_{0}$ and $\tau$ (these coordinates may be considered as fixed on the fluid element). The velocity of a fluid element is given in terms of the Lagrangian displacement $\boldsymbol{\xi}=\boldsymbol{r}-\boldsymbol{r}_{0}$ by $v=\partial \xi / \partial \tau$ (the velocity is defined at the position of the fluid element, which may be considered as a particle), and the Lagrangian form of the equation of continuity is 


$$
n J=n_{0}\left(\boldsymbol{r}_{0}, \tau=0\right) .
$$

The equation of conservation of entropy becomes

$$
\frac{\partial s}{\partial \tau}=0 .
$$

Introducing the Lagrange multipliers $a_{L}$ and $b_{L}$ (for each species), one has

$$
\begin{gathered}
\delta \int d^{3} r_{0} d \tau\left\{J \left[\sum_{q}\left(-\frac{n m c^{2}}{\gamma}-\frac{n k_{B} T}{\gamma_{A}-1}-n q \phi+n q \mathbf{A} \cdot \frac{\partial \xi}{\partial \tau}\right)\right.\right. \\
\left.\left.+\frac{1}{2}\left(\epsilon_{0} E^{2}-\frac{B^{2}}{\mu_{0}}\right)\right]+\sum_{q}\left(a_{L}\left(n J-n_{0}\right)+b_{L} \frac{\partial s}{\partial \tau}\right)\right\}=0 .
\end{gathered}
$$

The Euler-Lagrange equations are

(i) $\delta \phi \quad \nabla_{0} \cdot \boldsymbol{E}=\frac{1}{\epsilon_{0}} \sum_{q} \rho_{0}$,

(ii) $\delta \boldsymbol{A} \quad \nabla_{0} \times \boldsymbol{B}=\mu_{0} \sum_{q} \mathbf{j}_{0}+\frac{1}{c^{2}} \frac{\partial \boldsymbol{E}}{\partial \tau}$,

(iii) $\delta \xi \quad J n q\left[-\frac{\partial \phi}{\partial \xi}+\left(\frac{\partial \boldsymbol{A}}{\partial \xi}\right) \cdot \frac{\partial \xi}{\partial \tau}\right]$

$$
\begin{aligned}
= & \frac{\partial}{\partial \tau}\left\{J\left[n \gamma m c^{2}-n \gamma^{3} \frac{\partial}{\partial \gamma}\left(\frac{k_{B} T}{\gamma_{A}-1}\right)_{n, s}\right] \frac{1}{c^{2}} \frac{\partial \xi}{\partial \tau}+J n q \boldsymbol{A}\right\} \\
& +\nabla_{0} \cdot\left[\frac { \partial J } { \partial ( \nabla _ { 0 } \mathrm { r } ) } \left(-\frac{n m c^{2}}{\gamma}-\frac{n k_{B} T}{\gamma_{A}-1}-n q \phi\right.\right. \\
& \left.\left.+n q \boldsymbol{A} \cdot \frac{\partial \xi}{\partial \tau}+n a_{L}\right)\right]
\end{aligned}
$$

(iv) $\delta n \quad a_{L}=\frac{m c^{2}}{\gamma}+\frac{k_{B} T}{\gamma_{A}-1}+n \frac{\partial}{\partial n}\left(\frac{k_{B} T}{\gamma_{A}-1}\right)_{\gamma, s}+q \phi-q A \cdot \frac{\partial \xi}{\partial \tau}$,

(v) $\delta s \frac{\partial b_{L}}{\partial \tau}=-J n \frac{\partial}{\partial s}\left(\frac{k_{B} T}{\gamma_{A}-1}\right)_{n, \gamma}$.

Note that the variation of the electromagnetic field components with respect to the fluid displacements $\xi$ must be considered separately for each species and that $J$ must be different from zero so that the transformation $\boldsymbol{r}_{0} \rightleftarrows \boldsymbol{r}$ is one-to-one.

Now, the expression (iv) for $a_{L}$ can be substituted in the Euler-Lagrange equation (iii) for $\boldsymbol{\xi}$,

$$
\begin{aligned}
\frac{\partial}{\partial \tau}\left\{J\left[n \gamma m c^{2}-n \gamma^{3} \frac{\partial}{\partial \gamma}\left(\frac{k_{B} T}{\gamma_{A}-1}\right)_{n, s}\right] \frac{1}{c^{2}} \frac{\partial \boldsymbol{\xi}}{\partial \tau}+J n q \boldsymbol{A}\right\} \\
+\nabla_{0} \cdot\left[\frac{\partial J}{\partial\left(\nabla_{0} \boldsymbol{r}\right)} n^{2} \frac{\partial}{\partial n}\left(\frac{k_{B} T}{\gamma_{A}-1}\right)_{\gamma, s}\right] \\
=J n q\left[-\frac{\partial \phi}{\partial \boldsymbol{\xi}}+\left(\frac{\partial \boldsymbol{A}}{\partial \boldsymbol{\xi}}\right) \cdot \frac{\partial \boldsymbol{\xi}}{\partial \tau}\right] .
\end{aligned}
$$

The expressions for the Jacobian given in Appendix B give

$$
\frac{\partial J}{\partial\left(\nabla_{0} \boldsymbol{r}\right)}=\frac{\partial}{\partial\left(\nabla_{0} \boldsymbol{r}\right)}\left|\nabla_{0} \boldsymbol{r}\right|=\overline{\overline{\boldsymbol{C}}}=J\left[\left(\nabla_{0} \boldsymbol{r}\right)^{-1}\right]^{T},
$$

where $J=\left|\nabla_{0} \boldsymbol{r}\right|$ is the determinant of $\nabla_{0} \boldsymbol{r}$ and $\overline{\bar{C}}$ is the dyadic of the cofactors of the elements of $\nabla_{0} r$. Moreover, the formulas of transformation from Eulerian to Lagrangian coordinates, also derived in Appendix B, give

$$
\begin{aligned}
\boldsymbol{\nabla} \boldsymbol{r}_{0} & \equiv\left(\overline{\overline{\boldsymbol{I}}}+\nabla_{0} \xi\right)^{-1} \cdot \nabla_{0} \boldsymbol{r}_{0}=\left(\overline{\overline{\boldsymbol{I}}}+\nabla_{0} \xi\right)^{-1} \\
& =\left[\left(\overline{\overline{\boldsymbol{I}}}+\nabla_{0} \xi\right) \cdot \nabla \boldsymbol{r}\right]^{-1}=\left(\nabla_{0} \boldsymbol{r}\right)^{-1}
\end{aligned}
$$

Thus,

$$
\frac{\partial J}{\partial\left(\nabla_{0} \boldsymbol{r}\right)}=J\left(\nabla \boldsymbol{r}_{0}\right)^{T}
$$

It follows that

$$
\begin{aligned}
\boldsymbol{\nabla}_{0} \cdot & {\left[J\left(\nabla \boldsymbol{r}_{0}\right)^{T} n^{2} \frac{\partial}{\partial n}\left(\frac{k_{B} T}{\gamma_{A}-1}\right)_{\gamma, s}\right] } \\
= & \nabla_{0} \cdot\left[J\left(\nabla \boldsymbol{r}_{0}\right)^{T}\right] n^{2} \frac{\partial}{\partial n}\left(\frac{k_{B} T}{\gamma_{A}-1}\right)_{\gamma, s} \\
& +J\left(\nabla \boldsymbol{r}_{0}\right) \cdot \nabla_{0}\left[n^{2} \frac{\partial}{\partial n}\left(\frac{k_{B} T}{\gamma_{A}-1}\right)_{\gamma, s}\right] .
\end{aligned}
$$

But the first term on the right-hand side vanishes, since

$$
\nabla_{0} \cdot\left[J\left(\nabla r_{0}\right)^{T}\right]=\nabla_{0} \cdot \overline{\bar{C}}=0
$$

and the second term can be written as

$$
J\left(\nabla \boldsymbol{r}_{0}\right) \cdot \nabla_{0}\left[n^{2} \frac{\partial}{\partial n}\left(\frac{k_{B} T}{\gamma_{A}-1}\right)_{\gamma, s}\right]=J \nabla\left[n^{2} \frac{\partial}{\partial n}\left(\frac{k_{B} T}{\gamma_{A}-1}\right)_{\gamma, s}\right],
$$

so that the Euler-Lagrange equation for $\xi$ becomes

$$
\begin{aligned}
& \frac{\partial}{\partial \tau}\left\{J\left[n \gamma m c^{2}-n \gamma^{3} \frac{\partial}{\partial \gamma}\left(\frac{k_{B} T}{\gamma_{A}-1}\right)_{n, s}\right] \frac{1}{c^{2}} \frac{\partial \xi}{\partial \tau}+J n q A\right\} \\
& \quad+J \nabla\left[n^{2} \frac{\partial}{\partial n}\left(\frac{k_{B} T}{\gamma_{A}-1}\right)_{\gamma, s}\right]=J n q\left[-\nabla \phi+(\nabla \boldsymbol{A}) \cdot \frac{\partial \xi}{\partial \tau}\right] .
\end{aligned}
$$

The quantity $n J=n_{0}$ is independent of $\tau$; therefore,

$$
\begin{aligned}
\frac{\partial}{\partial \tau} & {\left[\gamma m \frac{\partial \xi}{\partial \tau}-\frac{\gamma^{3}}{c^{2}} \frac{\partial}{\partial \gamma}\left(\frac{k_{B} T}{\gamma_{A}-1}\right)_{n, s} \frac{\partial \xi}{\partial \tau}+q A\right] } \\
& =-\frac{1}{n} \nabla\left[n^{2} \frac{\partial}{\partial n}\left(\frac{k_{B} T}{\gamma_{A}-1}\right)_{\gamma, s}\right]-q \nabla \phi+q(\nabla A) \cdot \frac{\partial \boldsymbol{\xi}}{\partial \tau} .
\end{aligned}
$$

Using the thermodynamic relations,

$$
\begin{aligned}
& \frac{\partial}{\partial n}\left(\frac{k_{B} T}{\gamma_{A}-1}\right)_{\gamma, s}=\frac{k_{B} T}{n}, \\
& \frac{\partial}{\partial \gamma}\left(\frac{k_{B} T}{\gamma_{A}-1}\right)_{n, s}=-\frac{\gamma_{A}}{\gamma_{A}-1} \frac{k_{B} T}{\gamma},
\end{aligned}
$$

one obtains 


$$
\begin{aligned}
\frac{\partial}{\partial \tau} & {\left[\gamma m\left(1+\frac{\gamma_{A}}{\gamma_{A}-1} \frac{\gamma k_{B} T}{m c^{2}}\right) \frac{\partial \xi}{\partial \tau}+q A\right] } \\
& =-\frac{1}{n} \nabla\left(n k_{B} T\right)-q \nabla \phi+q(\nabla \boldsymbol{A}) \cdot \frac{\partial \xi}{\partial \tau},
\end{aligned}
$$

where the left-hand side corresponds to the time rate of change of the canonical momentum of the plasma fluid element. The transformation back to Eulerian coordinates is carried out by means of the following relations:

$$
\frac{\partial}{\partial \tau} \equiv \frac{\partial}{\partial t}+\boldsymbol{v} \cdot \boldsymbol{\nabla}, \quad \frac{\partial \boldsymbol{\xi}}{\partial \tau}=\boldsymbol{v}
$$

so that

$$
\begin{aligned}
& \left(\frac{\partial}{\partial t}+\boldsymbol{v} \cdot \boldsymbol{\nabla}\right)\left[\gamma\left(1+\frac{\gamma_{A}}{\gamma_{A}-1} \frac{\gamma k_{B} T}{m c^{2}}\right) m \boldsymbol{v}\right] \\
& =-\frac{1}{n} \nabla\left(n k_{B} T\right)-q \nabla \phi-q \dot{\boldsymbol{A}}-q \boldsymbol{v} \cdot \boldsymbol{\nabla} \boldsymbol{A}+q(\nabla \boldsymbol{A}) \cdot \boldsymbol{v}
\end{aligned}
$$

Multiplying by $n$ and using the equation of continuity, this equation becomes

$$
\begin{aligned}
\frac{\partial}{\partial t}[ & \left.\gamma\left(1+\frac{\gamma_{A}}{\gamma_{A}-1} \frac{\gamma k_{B} T}{m c^{2}}\right) n m \boldsymbol{v}\right] \\
& +\boldsymbol{\nabla} \cdot\left[\gamma\left(1+\frac{\gamma_{A}}{\gamma_{A}-1} \frac{\gamma k_{B} T}{m c^{2}}\right) n m \boldsymbol{v} \boldsymbol{v}+n k_{B} T \overline{\overline{\mathbf{I}}}\right] \\
= & -n q \nabla \phi-n q \dot{\boldsymbol{A}}+n q \boldsymbol{v} \times(\boldsymbol{\nabla} \times \boldsymbol{A}),
\end{aligned}
$$

which recovers the equation of motion in the Eulerian form. This can be easily verified using the definitions $\rho=n q, \boldsymbol{j}=n q \boldsymbol{v}, \boldsymbol{E}=-\boldsymbol{\nabla} \phi$ $-\partial \boldsymbol{A} / \partial t$, and $\boldsymbol{B}=\boldsymbol{\nabla} \times \boldsymbol{A}$, and the equation of state $p=n k_{B} T$, which gives back Eq. (49).

\section{CLEBSCH REPRESENTATION} form

Recall the Euler-Lagrange equation (iii) for $\delta \boldsymbol{v}$ in the Clebsch

$$
m \alpha \boldsymbol{v}+q \boldsymbol{A}=\boldsymbol{\nabla} a-b \nabla s-\left(\nabla \boldsymbol{r}_{0}\right) \cdot \boldsymbol{c} .
$$

The Jacobian dyadic of the transformation $\boldsymbol{r}=\boldsymbol{r}\left(\boldsymbol{r}_{0}, t\right)$ from the initial position $\boldsymbol{r}_{0}$ to the present position $\boldsymbol{r}$ is $\overline{\overline{\boldsymbol{J}}}=\partial \boldsymbol{r} / \partial \boldsymbol{r}_{0}=\nabla_{0} \boldsymbol{r}$, so that $\nabla \boldsymbol{r}_{0}=\overline{\overline{\boldsymbol{J}}}^{-1}$ produces the inverse transformation $\boldsymbol{r}_{0}=\boldsymbol{r}_{0}(\boldsymbol{r}, t)$ from the present to the initial position. In this way, $\left(\nabla \boldsymbol{r}_{0}\right) \cdot \boldsymbol{c}=\boldsymbol{c}_{0}$ corresponds to the initial value of the Lagrange multiplier $c$, which satisfies the equation of motion $d c / d t=0$. Defining a reference value $\boldsymbol{P}_{0}=-\boldsymbol{c}_{0}$, the canonical momentum of species $q$ is given by

$$
\boldsymbol{P}=m \alpha \boldsymbol{v}+q \boldsymbol{A}=\boldsymbol{P}_{0}+\nabla a-b \boldsymbol{\nabla} s .
$$

Since the vector potential can be modified by a gauge transformation, this reference value is somewhat arbitrary. In particular, if $\nabla a=b \nabla s$ at some point, the reference value $\boldsymbol{P}_{0}$ corresponds to the initial value of the canonical momentum at this point. Hence, $\boldsymbol{P}_{0}$ can be considered as the initial value of $\boldsymbol{P}$ within a convenient gauge transformation. A canonical vorticity vector $\Omega$ can be defined by

$$
\Omega=\nabla \times \boldsymbol{P}=\boldsymbol{\nabla} \times \boldsymbol{P}_{0}-\boldsymbol{\nabla} \times(b \boldsymbol{\nabla})=\Omega_{0}+\nabla s \times \nabla b,
$$

where $\Omega_{0}=\nabla \times \boldsymbol{P}_{0}$ is the reference vorticity associated with the Lin multiplier $\boldsymbol{c}_{0}=-\boldsymbol{P}_{0}$. Note that the canonical vorticity is not affected by the longitudinal Euler potential. The canonical momentum can also be written as

$$
\begin{aligned}
\boldsymbol{P} & =m \alpha \boldsymbol{v}+q \boldsymbol{A}=\boldsymbol{P}_{0}+\boldsymbol{\nabla}(a-s b)+s \boldsymbol{\nabla} b \\
& =\boldsymbol{P}_{0}+\boldsymbol{\nabla} \lambda+s \boldsymbol{\nabla} b,
\end{aligned}
$$

where $\lambda=a-s b$ denotes a new longitudinal Clebsch potential, without changing the canonical vorticity,

$$
\Omega=\Omega_{0}+\nabla s \times \nabla b .
$$

The canonical vorticity includes flow vorticity and magnetic field values. The parameters $s$ and $b$ are the Euler potentials in the Clebsch representation

$$
\left\{\begin{array}{l}
\left(\Omega-\Omega_{0}\right) \cdot \nabla s=0, \\
\left(\Omega-\Omega_{0}\right) \cdot \nabla b=0,
\end{array}\right.
$$

with $\nabla \cdot \Omega=0$ and $\nabla \cdot \Omega_{0}=0$. The canonical vorticity increment $\Omega-\Omega_{0}$ lies in the intersection of the two surfaces defined by $s=$ constant and $b=$ constant, and $\left(\Omega-\Omega_{0}\right) \cdot \nabla \lambda$ gives the triple product,

$$
\left(\Omega-\Omega_{0}\right) \cdot \nabla \lambda=\nabla a \cdot \nabla b \times \nabla s .
$$

The evolution of the potentials $s$ and $b$ is governed by the equations of motion,

$$
\left\{\begin{array}{l}
\frac{d s}{d t}=0 \\
\frac{d b}{d t}=-\frac{\partial}{\partial s}\left(\frac{k_{B} T}{\gamma_{A}-1}\right)_{n, \gamma}=-T .
\end{array}\right.
$$

The advection of the thermal potential $b$ depends on the variation with $s$ of the thermal energy density for constant $n$ and $\gamma$, which is simply proportional to the temperature $T$ of the perfect fluid. Note that the motion of the longitudinal potential $\lambda$ is governed by the following equation:

$$
\frac{d \lambda}{d t}=\frac{d a}{d t}+T s
$$

The Euler potentials are not unique since an arbitrary function of $s$ can be added to $b$, or vice versa (but not simultaneously). Indeed, add$\operatorname{ing} f(s)$ to $b$ gives

$$
\nabla s \times \nabla(b+f(s))=\nabla s \times \nabla b+\nabla s \times\left(\frac{\partial f}{\partial s} \nabla s\right)=\nabla s \times \nabla b .
$$

The specific helicity associated with $\boldsymbol{P}$ is given by

$$
\begin{aligned}
\boldsymbol{P} \cdot \Omega & =\left(\boldsymbol{P}_{0}+\nabla a-b \nabla s\right) \cdot\left(\Omega_{0}+\nabla s \times \nabla b\right) \\
& =\boldsymbol{P}_{0} \cdot\left(2 \Omega-\Omega_{0}\right)+\nabla \cdot\left(a \Omega+b \nabla s \times \boldsymbol{P}_{0}\right),
\end{aligned}
$$

and the total helicity is given by the volume integral

$$
\begin{aligned}
K= & \int_{V}(\boldsymbol{P} \cdot \Omega) d^{3} r=\int_{V} \boldsymbol{P}_{0} \cdot\left(2 \Omega-\Omega_{0}\right) d^{3} r \\
& +\oint_{S}\left(a \Omega+b \boldsymbol{\nabla} s \times \boldsymbol{P}_{0}\right) \cdot d^{2} \boldsymbol{r} .
\end{aligned}
$$

The first term in the right-hand side corresponds to the evolution of the total helicity inside the volume $V$. The second term gives the flux 
of both the canonical vorticity $\Omega$, weighted by the longitudinal Lagrangian coefficient $a$, and $\boldsymbol{\nabla} \times \times \boldsymbol{P}_{0}$, weighted by the Lagrangian coefficient $b$, passing through the surface bounding the volume $V$. The longitudinal coefficient $a$ of the canonical momentum is associated with the conservation of fluid elements (particles), and the thermal coefficient $b$ is associated with the conservation of energy. The surface term vanishes if $a$ and $b$ are continuous and both the canonical vorticity $\Omega$ and $\nabla s \times \boldsymbol{P}_{0}$ are tangent to the surface $S$. The Clebsch coefficients $a$ and $b$ may present singularities inside the volume $V$ or on the surface $S$. Since helicity measures the linkage or knottedness of the vortex lines in a flow, ${ }^{17}$ the internal singularities may be associated with small regions of increased diffusivity and $X$-points (knots) of the $\Omega$ field. The surface singularities are usually associated with external sources of $\Omega$ flux. For example, helicity can be injected in a plasma by discontinuities in the potentials (voltage drops on electrodes inserted in the plasma). Another classic example is given by Woltjer's theorem that uses the invariance of the magnetic helicity in an ideal plasma. ${ }^{19,23}$ These effects show the importance of surface terms in the Clebsch representation. Note that the magnetic field $\boldsymbol{B}=\boldsymbol{\nabla} \times \boldsymbol{A}$, modified by the (large) charge to mass ratio $q / m$, has the same role as the fluid vorticity $\omega=\nabla \times(\alpha \boldsymbol{v})$, modified by the relativistic inertia factor $\alpha$. In terms of the longitudinal potential $\lambda$, the specific helicity becomes

$$
\boldsymbol{P} \cdot \Omega=\boldsymbol{P}_{0} \cdot\left(2 \Omega-\Omega_{0}\right)+\boldsymbol{\nabla} \cdot\left(\lambda \Omega-s \boldsymbol{\nabla} b \times \boldsymbol{P}_{0}\right) .
$$

Using the transport theorem (cf. Appendix B), the time variation of the total helicity is given by

$$
\frac{d K}{d t}=\frac{\partial}{\partial t} \int_{V}(\boldsymbol{P} \cdot \Omega) d^{3} r+\oint_{S}(\boldsymbol{P} \cdot \Omega) \boldsymbol{v} \cdot d^{2} \boldsymbol{r}
$$

This shows that, in steady state $(\partial / \partial t \equiv 0)$, the total helicity $K$ is invariant if $\boldsymbol{v}$ is tangent to the surface $S$, generalizing Woltjer's theorem. ${ }^{19}$

If $S$ is an open surface bounded by the contour $C$ formed by the line elements $d \ell$, the $\Omega$ flux through $S$ is given according to Stokes theorem by

$$
\begin{aligned}
\Psi & =\int_{S} \Omega \cdot d^{2} \boldsymbol{r}=\int_{S}(\boldsymbol{\nabla} \times \boldsymbol{P}) \cdot d^{2} \boldsymbol{r} \\
& =\oint_{C} \boldsymbol{P} \cdot d \boldsymbol{\ell}=\oint_{C}\left(\boldsymbol{P}_{0}+\nabla a-b \boldsymbol{\nabla} s\right) \cdot d \boldsymbol{\ell} \\
& =a\left(C_{2}\right)-a\left(C_{1}\right)+\oint_{C}\left(\boldsymbol{P}_{0}-b \boldsymbol{\nabla} s\right) \cdot d \boldsymbol{\ell} .
\end{aligned}
$$

The integral of the conservative vector field $\nabla a$ depends only on the values of $a$ on the endpoints of the path $C$. This gives a vanishing contribution if $a$ does not have a singularity along the closed contour $C$. Returning to the previous example, a voltage drop across close isolated electrodes inserted into a magnetized plasma (idealized divertors) can produce a finite vorticity flux. The line integral of the reference value $\boldsymbol{P}_{0}$ of the canonical momentum corresponds to the circulation,

$$
\Gamma_{0}=\oint_{C} \boldsymbol{P}_{0} \cdot d \boldsymbol{\ell}
$$

Thus,

$$
\Psi=\Gamma_{0}+a\left(C_{2}\right)-a\left(C_{1}\right)-\oint_{C} b \nabla s \cdot d \ell .
$$

The circulation $\Gamma_{0}$ may include the effect of non-conservative sources, such as the electromotive force of transformer action. This electromotive force is responsible, for example, for the inductive helicity injection in a tokamak. In general, the increment $a\left(C_{2}\right)-a\left(C_{1}\right)$ can be included in $\Gamma_{0}$, although they denote different vorticity sources. Assuming continuous values of $a$, the flux through a surface $S_{s}$ bounded by the closed contour $C_{s}$, which is defined on the Euler surface $s$, becomes

$$
\Psi_{s}=\Gamma_{0}-\oint_{C_{s}} b \nabla s \cdot d \ell(s)
$$

The flux calculation simplifies for a $\Omega$ field configuration in which $s$ is an ignorable quantity, that is, the system is homogeneous with respect to $s$. In this case, the Euler potential $b$ is independent of the symmetry quantity $s$, and the flux $\Psi_{s}$ becomes

$$
\Psi_{s}=\Gamma_{0}-b \oint_{C_{s}} d s
$$

Defining a normalized flux $\psi_{s}=\left(\Psi_{s}-\Gamma_{0}\right) / \oint d s$, the Euler potential $b$ is given by $b=-\psi$ s so that the corresponding canonical momentum $\boldsymbol{P}$ and canonical vorticity $\Omega$ components are given by

$$
\left\{\begin{array}{l}
\boldsymbol{P}_{s}=\boldsymbol{P}_{0}+\nabla a+\psi_{s} \nabla s, \\
\Omega_{s}=\nabla \times \boldsymbol{P}_{s}=\Omega_{0}+\nabla \psi_{s} \times \nabla s .
\end{array}\right.
$$

If one component of the field $\Omega$ lies in a symmetry direction, the total field can be written as a sum in terms of the reference value and of the flux associated with the symmetry component. These simplified expressions are restricted to the case in which the system is homogeneous with respect to $s$, but gives some idea about the meaning of the Euler potential $b$, at least over a surface of nearly homogeneous specific entropy.

Given the canonical momentum in the following form (here repeated for convenience):

$$
\boldsymbol{P}=m \alpha \boldsymbol{v}+q \boldsymbol{A}=\boldsymbol{P}_{0}+\nabla a-b \boldsymbol{\nabla},
$$

recall that the Lagrangian coefficient $a$ satisfies the equation of motion (Euler-Lagrange equation (iv) for $\delta n$ in the Eulerian formulation),

$$
\frac{d a}{d t}=-\frac{m c^{2}}{\gamma}-\frac{k_{B} T}{\gamma_{A}-1}-n \frac{\partial}{\partial n}\left(\frac{k_{B} T}{\gamma_{A}-1}\right)_{\gamma, s}-q \phi+q \boldsymbol{v} \cdot \boldsymbol{A} .
$$

Using Maxwell's differential relation,

$$
\frac{\partial}{\partial n}\left(\frac{k_{B} T}{\gamma_{A}-1}\right)_{\gamma, s}=\frac{k_{B} T}{n}
$$

one can write a set of equations of motion for the Euler coefficients of the Clebsch representation for $\boldsymbol{P}$,

$$
\left\{\begin{array}{l}
\frac{1}{k_{B}} \frac{d s}{d t}=\frac{1}{T} \frac{d}{d t}\left(\frac{T}{\gamma_{A}-1}\right)+\frac{\gamma_{A}}{\gamma_{A}-1} \frac{1}{\gamma} \frac{d \gamma}{d t}-\frac{1}{n} \frac{d n}{d t}=0, \\
\frac{d a}{d t}=-\frac{m c^{2}}{\gamma}-\frac{\gamma_{A} k_{B} T}{\gamma_{A}-1}-q \phi+q v \cdot A, \\
\frac{d b}{d t}=-T, \\
\frac{d c}{d t}=0,
\end{array}\right.
$$


where

$$
\boldsymbol{c}=\overline{\overline{\boldsymbol{J}}} \cdot \boldsymbol{c}_{0}=-\overline{\overline{\boldsymbol{J}}} \cdot \boldsymbol{P}_{0},
$$

and $\overline{\overline{\boldsymbol{J}}}=\partial \boldsymbol{r} / \partial \boldsymbol{r}_{0}=\nabla_{0} \boldsymbol{r}=\overline{\overline{\boldsymbol{I}}}+\nabla_{0} \boldsymbol{\xi}$ is the Jacobian dyadic ( $\boldsymbol{\xi}$ is the Lagrangian displacement and $\boldsymbol{v}=d \boldsymbol{\xi} / d t$ ). Hence, the present value of the canonical momentum $\boldsymbol{P}$ depends not only of the evolution of the mean fluid variables but also of the reference (initial) distribution $\boldsymbol{P}_{0}$, that is, of the trajectory of each fluid element. In this way, the Clebsch representation is equivalent to the Lagrangian approach (cf. Subsection II C), which describes the motion of each fluid element. In the Eulerian formulation, the substitution of the equations of motion for the Euler coefficients in the expression of the canonical momentum leads to an equation for $d \boldsymbol{P} / d t$ which depends only on the mean fluid variables (cf. Subsection II B). As stated in the Introduction, the two formulations are equivalent.

\section{A HYDROMAGNETIC CAUCHY INVARIANT}

Making use of the vector relation,

$$
(\boldsymbol{A} \cdot \boldsymbol{\nabla}) \boldsymbol{A}=(\boldsymbol{\nabla} \times \boldsymbol{A}) \times \boldsymbol{A}+\frac{1}{2} \nabla(\boldsymbol{A} \cdot \boldsymbol{A}),
$$

applied to the vector $\alpha \boldsymbol{v}$, the acceleration can be written in the following form:

$$
\begin{aligned}
\frac{d}{d t}(\alpha \boldsymbol{v}) & =\frac{\partial}{\partial t}(\alpha \boldsymbol{v})+\boldsymbol{v} \cdot \boldsymbol{\nabla}(\alpha \boldsymbol{v}) \\
& =\frac{\partial}{\partial t}(\alpha \boldsymbol{v})+(\boldsymbol{\nabla} \times \alpha \boldsymbol{v}) \times \boldsymbol{v}+\frac{\boldsymbol{\nabla}(\alpha v)^{2}}{2 \alpha},
\end{aligned}
$$

and the curl gives, using the continuity equation,

$$
\begin{aligned}
\boldsymbol{\nabla} \times\left(\frac{d}{d t}(\alpha \boldsymbol{v})\right)= & \frac{\partial}{\partial t}[\boldsymbol{\nabla} \times(\alpha \boldsymbol{v})]+\boldsymbol{\nabla} \times[(\boldsymbol{\nabla} \times \alpha \boldsymbol{v}) \times \boldsymbol{v}]+\left(\frac{\nabla v^{2}}{2}\right) \times \nabla \alpha \\
= & \frac{d}{d t}[\boldsymbol{\nabla} \times(\alpha \boldsymbol{v})]-\frac{(\boldsymbol{\nabla} \times \alpha \boldsymbol{v})}{n} \frac{d n}{d t} \\
& -[(\boldsymbol{\nabla} \times \alpha \boldsymbol{v}) \cdot \boldsymbol{\nabla}] \boldsymbol{v}+\left(\frac{\boldsymbol{\nabla} v^{2}}{2}\right) \times \nabla \alpha
\end{aligned}
$$

Thus,

$$
\begin{aligned}
\frac{d}{d t}\left(\frac{\boldsymbol{\nabla} \times(\alpha \boldsymbol{v})}{n}\right)= & \left(\frac{\boldsymbol{\nabla} \times(\alpha \boldsymbol{v})}{n} \cdot \boldsymbol{\nabla}\right) \boldsymbol{v} \\
& +\frac{1}{n} \boldsymbol{\nabla} \times\left(\frac{d}{d t}(\alpha \boldsymbol{v})\right)-\frac{\left(\boldsymbol{\nabla} v^{2}\right) \times \nabla \alpha}{2 n} .
\end{aligned}
$$

Using the second Maxwell differential relation in (40), the relativistic inertia factor defined in Eq. (32) becomes

$$
\alpha=\gamma\left(1+\frac{\gamma_{A}}{\gamma_{A}-1} \frac{\gamma k_{B} T}{m c^{2}}\right)
$$

Hence,

$$
\nabla \alpha=\frac{\partial \alpha}{\partial v} \nabla v+\frac{\partial \alpha}{\partial T} \nabla T
$$

where

$$
\left\{\begin{array}{l}
\frac{\partial \alpha}{\partial v}=\left(1+\frac{\gamma_{A}}{\gamma_{A}-1} \frac{2 \gamma k_{B} T}{m c^{2}}\right) \frac{d \gamma}{d v}, \\
\frac{\partial \alpha}{\partial T}=\frac{\gamma_{A}}{\gamma_{A}-1} \frac{\gamma^{2} k_{B} T}{m c^{2}}\left(\frac{1}{T}-\frac{d \gamma_{A} / d T}{\gamma_{A}\left(\gamma_{A}-1\right)}\right) .
\end{array}\right.
$$

Taking into account the transformation of the absolute temperature $T=\stackrel{\circ}{T} / \gamma$ from the frame moving with the fluid velocity $\boldsymbol{v}$ to the rest frame temperature $\stackrel{T}{T}$ (cf. Appendix A), this expression becomes

$$
\nabla \alpha=\left(\frac{\partial \alpha}{\partial v}-\frac{\partial \alpha}{\partial T} \frac{\gamma^{2} v T}{c^{2}}\right) \nabla v \Rightarrow \nabla v \times \nabla \alpha=0 .
$$

This leads to a diffusion equation for the vorticity,

$$
\frac{d}{d t}\left(\frac{\boldsymbol{\nabla} \times(\alpha \boldsymbol{v})}{n}\right)=\left(\frac{\boldsymbol{\nabla} \times(\alpha \boldsymbol{v})}{n} \cdot \boldsymbol{\nabla}\right) \boldsymbol{v}+\frac{1}{n} \boldsymbol{\nabla} \times\left(\frac{d}{d t}(\alpha \boldsymbol{v})\right) .
$$

Now, the equation of momentum conservation,

$$
\frac{d}{d t}(m \alpha \boldsymbol{v})=-\frac{\nabla p}{n}+q(\boldsymbol{E}+\boldsymbol{v} \times \boldsymbol{B}),
$$

gives

$$
\begin{aligned}
\boldsymbol{\nabla} \times\left(\frac{d}{d t}(m \alpha \boldsymbol{v})\right)= & -\frac{\boldsymbol{\nabla} p \times \boldsymbol{\nabla} n}{n^{2}}+q \boldsymbol{\nabla} \times \boldsymbol{E} \\
& +q[-\boldsymbol{B}(\boldsymbol{\nabla} \cdot \boldsymbol{v})+(\boldsymbol{B} \cdot \boldsymbol{\nabla}) \boldsymbol{v}-(\boldsymbol{v} \cdot \boldsymbol{\nabla}) \boldsymbol{B}] .
\end{aligned}
$$

With the help of the continuity equation and Faraday's law,

$$
\boldsymbol{\nabla} \times\left(\frac{d}{d t}(m \alpha \boldsymbol{v})\right)=-\frac{\nabla p \times \nabla n}{n^{2}}-q \frac{d \boldsymbol{B}}{d t}+q \frac{\boldsymbol{B}}{n} \frac{d n}{d t}+q(\boldsymbol{B} \cdot \boldsymbol{\nabla}) \boldsymbol{v} .
$$

Substituting the vorticity diffusion equation,

$$
\frac{d}{d t}\left(\frac{\boldsymbol{\nabla} \times(m \alpha \boldsymbol{v})+q \boldsymbol{B}}{n}\right)=\left(\frac{\boldsymbol{\nabla} \times(m \alpha \boldsymbol{v})+q \boldsymbol{B}}{n} \cdot \boldsymbol{\nabla}\right) \boldsymbol{v}-\frac{\boldsymbol{\nabla} p \times \boldsymbol{\nabla} n}{n^{3}} .
$$

The perfect fluid flow is barotropic (in which the pressure $p$ and the density $n$ are directly related), so that the last term in the right-hand side vanishes. The canonical vorticity $\Omega$ defined in Sec. III reads

$$
\Omega=\nabla \times \boldsymbol{P}=\boldsymbol{\nabla} \times(m \alpha \boldsymbol{v})+q \boldsymbol{B},
$$

so that the diffusion equation for $\Omega$ becomes

$$
\frac{d}{d t}\left(\frac{\Omega}{n}\right)=\left(\frac{\Omega}{n} \cdot \nabla\right) v .
$$

Introducing a change in the dependent variables such that ${ }^{2}$

$$
\frac{\Omega}{n}=C \cdot \nabla_{0} r
$$

the canonical vorticity equation becomes

$$
\frac{d}{d t}\left(\boldsymbol{C} \cdot \nabla_{0} \boldsymbol{r}\right)=\left[\left(\boldsymbol{C} \cdot \nabla_{0} \boldsymbol{r}\right) \cdot \boldsymbol{\nabla}\right] \boldsymbol{v} .
$$

Here, $\nabla_{0} \boldsymbol{r}=\partial \boldsymbol{r} / \partial \boldsymbol{r}_{0}=\overline{\overline{\boldsymbol{J}}}$ is the Jacobian dyadic of the transformation $\boldsymbol{r}=\boldsymbol{r}\left(\boldsymbol{r}_{0}, t\right)$ from the Lagrangian $\boldsymbol{r}_{0}$ to the Eulerian $\boldsymbol{r}$ coordinates 
$\left(|\overline{\overline{\boldsymbol{J}}}| \neq 0\right.$ and $\left.\nabla_{0} \equiv \overline{\overline{\boldsymbol{J}}} \cdot \nabla\right)$. The transformation $\boldsymbol{r}=\boldsymbol{r}\left(\boldsymbol{r}_{0}, t\right)$ specifies the trajectory of a material particle (or fluid element, cf. Appendix B). For fixed $t$, it determines the transformation of the particle from the initial position $\boldsymbol{r}_{0}$ to the position $\boldsymbol{r}$ at time $t$. Since

$$
\begin{aligned}
\frac{d}{d t}\left(\boldsymbol{C} \cdot \nabla_{0} \boldsymbol{r}\right) & =\frac{d \boldsymbol{C}}{d t} \cdot \nabla_{0} \boldsymbol{r}+\boldsymbol{C} \cdot \nabla_{0} \boldsymbol{v} \\
& =\frac{d \boldsymbol{C}}{d t} \cdot \nabla_{0} \boldsymbol{r}+\boldsymbol{C} \cdot \overline{\overline{\boldsymbol{J}}} \cdot \boldsymbol{\nabla} \boldsymbol{v} \\
& =\frac{d \boldsymbol{C}}{d t} \cdot \nabla_{0} \boldsymbol{r}+\left(\boldsymbol{C} \cdot \nabla_{0} \boldsymbol{r}\right) \cdot \boldsymbol{\nabla} \boldsymbol{v},
\end{aligned}
$$

the canonical vorticity equation reduces to

$$
\begin{aligned}
\frac{d \boldsymbol{C}}{d t} \cdot \nabla_{0} \boldsymbol{r}=0 & \Rightarrow \frac{d \boldsymbol{C}}{d t}=0 \\
& \Rightarrow \boldsymbol{C}=\boldsymbol{C}\left(\boldsymbol{r}_{0}\right) .
\end{aligned}
$$

Thus,

$$
\frac{\Omega}{n}=C\left(r_{0}\right) \cdot \nabla_{0} r
$$

Setting $t=0$,

$$
\frac{\Omega}{n}=\frac{\Omega_{0}}{n_{0}} \cdot \nabla_{0} r
$$

This result was obtained, for an incompressible fluid and without the magnetic field, by Cauchy in $1815 .{ }^{16,18}$ Cauchy demonstrated that a fluid element that is in irrotational motion initially remains in this condition throughout the flow. However, the hydromagnetic Cauchy invariant shows that the magnetic field may introduce flow vorticity in an otherwise irrotational motion. This has important implications in the rotation curve of galaxies and in the mass accretion of astrophysical systems, where the gravitomagnetic field replaces the magnetic field.

\section{HAMILTONIAN DENSITY AND THE ENERGY INTEGRAL}

The momentum densities that canonically conjugate to the Lagrangian field coordinates $\phi, \boldsymbol{A}, \boldsymbol{\xi}, n$, and $s$ are

$$
\left\{\begin{array}{l}
\pi_{\phi}=0 \\
\pi_{A}=-\epsilon_{0} \boldsymbol{E}, \\
\pi_{\xi}=n_{0} \gamma m\left(1+\frac{\gamma_{A}}{\gamma_{A}-1} \frac{\gamma k_{B} T}{m c^{2}}\right) \frac{\partial \xi}{\partial \tau}+n_{0} q \boldsymbol{A}, \\
\pi_{n}=0 \\
\pi_{s}=b .
\end{array}\right.
$$

Using the Lagrangian density,

$$
\begin{aligned}
\mathcal{L}_{0}= & \sum_{q}\left(-\frac{n_{0} m c^{2}}{\gamma}-\frac{n_{0} k_{B} T}{\gamma_{A}-1}-n_{0} q \phi+n_{0} q \mathbf{A} \cdot \frac{\partial \xi}{\partial \tau}\right) \\
& +\frac{1}{2}\left(\epsilon_{0} E^{2}-\frac{B^{2}}{\mu_{0}}\right),
\end{aligned}
$$

the Hamiltonian density in Lagrangian coordinates can be easily calculated

$$
\begin{aligned}
\mathcal{H}_{0}= & \sum_{\alpha} \pi_{\alpha} \frac{\partial \varphi_{\alpha}}{\partial \tau}-\mathcal{L}_{0}=-\epsilon_{0} \boldsymbol{E} \cdot \frac{\partial \boldsymbol{A}}{\partial \tau} \\
& +\sum_{q}\left[n_{0} \gamma m\left(1+\frac{\gamma_{A}}{\gamma_{A}-1} \frac{\gamma k_{B} T}{m c^{2}}\right)\left(\frac{\partial \xi}{\partial \tau}\right)^{2}+n_{0} q \boldsymbol{A} \cdot \frac{\partial \xi}{\partial \tau}\right] \\
& -\left[\sum_{q}\left(-\frac{n_{0} m c^{2}}{\gamma}-\frac{n_{0} k_{B} T}{\gamma_{A}-1}-n_{0} q \phi+n_{0} q \boldsymbol{A} \cdot \frac{\partial \xi}{\partial \tau}\right)\right. \\
& \left.+\frac{1}{2}\left(\epsilon_{0} E^{2}-\frac{B^{2}}{\mu_{0}}\right)\right] \\
= & \sum_{q}\left[n_{0} \gamma m c^{2}+\frac{n_{0} k_{B} T}{\gamma_{A}-1}\left(1+\frac{\gamma_{A} \beta^{2}}{1-\beta^{2}}\right)+n_{0} q \phi\right] \\
& -\epsilon_{0} \boldsymbol{E} \cdot \frac{\partial \boldsymbol{A}}{\partial \tau}-\frac{1}{2}\left(\epsilon_{0} E^{2}-\frac{B^{2}}{\mu_{0}}\right) .
\end{aligned}
$$

Recalling that $n_{0}=n J$, the Hamiltonian density can be defined in the Eulerian sense by

$$
\begin{aligned}
\mathcal{H}= & \sum_{q}\left[n \gamma m c^{2}+\frac{n k_{B} T}{\gamma_{A}-1}\left(1+\frac{\gamma_{A} \beta^{2}}{1-\beta^{2}}\right)+n q \phi\right] \\
& -\epsilon_{0} \boldsymbol{E} \cdot \frac{\partial \boldsymbol{A}}{\partial \tau}-\frac{1}{2}\left(\epsilon_{0} E^{2}-\frac{B^{2}}{\mu_{0}}\right)
\end{aligned}
$$

so that

$$
H=\int d^{3} r_{0} J \mathcal{H}_{0}=\int d^{3} r \mathcal{H}
$$

Note that, although the same graphical symbol was used, the electromagnetic field variables in Eulerian and Lagrangian coordinates correspond to energy densities related by the ratio $n / n_{0}$. The electromagnetic field Hamiltonian density can also be written in the following form:

$$
\begin{aligned}
\mathcal{H}_{e m} & =-\epsilon_{0} \boldsymbol{E} \cdot \frac{\partial \boldsymbol{A}}{\partial \tau}-\frac{1}{2}\left(\epsilon_{0} E^{2}-\frac{B^{2}}{\mu_{0}}\right) \\
& =\epsilon_{0} \boldsymbol{E} \cdot \nabla \phi+\frac{1}{2}\left(\epsilon_{0} E^{2}+\frac{B^{2}}{\mu_{0}}\right) .
\end{aligned}
$$

Hence, using Gauss's law,

$$
\begin{aligned}
\mathcal{H}= & \sum_{q}\left[n \gamma m c^{2}+\frac{n k_{B} T}{\gamma_{A}-1}\left(1+\frac{\gamma_{A} \beta^{2}}{1-\beta^{2}}\right)\right] \\
& +\epsilon_{0} \nabla \cdot(\phi \boldsymbol{E})+\frac{1}{2}\left(\epsilon_{0} E^{2}+\frac{B^{2}}{\mu_{0}}\right) .
\end{aligned}
$$

According to Sec. II A, the energy integral is given by

$$
\begin{aligned}
\frac{d H}{d t}= & \frac{d}{d t}\left\{\left\{\sum_{q}\left[n \gamma m c^{2}+\frac{n k_{B} T}{\gamma_{A}-1}\left(1+\frac{\gamma_{A} \beta^{2}}{1-\beta^{2}}\right)\right]\right.\right. \\
& \left.+\frac{1}{2}\left(\epsilon_{0} E^{2}+\frac{B^{2}}{\mu_{0}}\right)\right\} d^{3} r+\frac{d}{d t} \oint \epsilon_{0} \phi \boldsymbol{E} \cdot d^{2} \boldsymbol{r}=0 .
\end{aligned}
$$


The stability of the fluid plasma can be investigated introducing a Lagrangian perturbation $\xi$ in the energy integral. ${ }^{22}$

\section{CONSERVATION EQUATIONS}

The conservation equations can be written in a compact form introducing the energy and momentum densities, and the stress tensor for each species of the plasma fluid

$$
\begin{aligned}
& U_{f}=\gamma n m c^{2}+\left(\frac{1}{\gamma_{A}-1}+\beta^{2}\right) \gamma^{2} p \quad \text { fluid energy density, } \\
& \boldsymbol{G}_{f}=\left(\gamma n m+\frac{\gamma_{A}}{\gamma_{A}-1} \frac{\gamma^{2} p}{c^{2}}\right) \boldsymbol{v} \quad \text { fluid momentum density, } \\
& \overline{\overline{\boldsymbol{T}}}_{f}=\gamma n m \boldsymbol{v} \boldsymbol{v}+\left(\overline{\overline{\boldsymbol{I}}}+\frac{\gamma_{A}}{\gamma_{A}-1} \gamma^{2} \frac{\boldsymbol{v} \boldsymbol{v}}{c^{2}}\right) p \quad \text { material stress tensor. }
\end{aligned}
$$

Hence, the momentum and energy conservation equations become

$$
\sum_{q}\left(\frac{\partial \boldsymbol{G}_{f}}{\partial t}+\nabla \cdot \overline{\overline{\boldsymbol{T}}}_{f}\right)=\sum_{q}(\rho \boldsymbol{E}+\boldsymbol{j} \times \boldsymbol{B})
$$

and

$$
\sum_{q}\left(\frac{\partial U_{f}}{\partial t}+c^{2} \nabla \cdot \boldsymbol{G}_{f}\right)=\sum_{q}(\boldsymbol{j} \cdot \boldsymbol{E}) .
$$

Note the following relations:

$$
\begin{aligned}
\overline{\overline{\boldsymbol{T}}}_{f}: \overline{\overline{\boldsymbol{I}}} & =\gamma n m v^{2}+\left(3+\frac{\gamma_{A}}{\gamma_{A}-1} \gamma^{2} \beta^{2}\right) p \\
\overline{\overline{\boldsymbol{T}}}_{f}: \frac{\boldsymbol{v} \boldsymbol{v}}{v^{2}} & =\gamma n m v^{2}+\left(1+\frac{\gamma_{A}}{\gamma_{A}-1} \gamma^{2} \beta^{2}\right) p=\beta^{2} U_{f}+\left(1+\beta^{2}\right) p, \\
2 p & =\overline{\overline{\boldsymbol{T}}}_{f}:\left(\overline{\overline{\boldsymbol{I}}}-\frac{v v}{v^{2}}\right), \\
\beta^{2} U_{f} & =\overline{\overline{\boldsymbol{T}}}_{f}: \frac{v v}{v^{2}}-\left(\frac{1+\beta^{2}}{2}\right) \overline{\overline{\boldsymbol{T}}}_{f}:\left(\overline{\overline{\boldsymbol{I}}}-\frac{v v}{v^{2}}\right) .
\end{aligned}
$$

Using Gauss's, Ampère's, and Faraday's laws, the momentum and energy conservation equations can be written as

$$
\begin{aligned}
\frac{\partial}{\partial t}\left(\sum_{q} \boldsymbol{G}_{f}+\boldsymbol{G}_{e m}\right)+\nabla \cdot\left(\sum_{q} \overline{\overline{\boldsymbol{T}}}_{f}+\overline{\overline{\boldsymbol{T}}}_{e m}\right) & =0, \\
\frac{\partial}{\partial t}\left(\sum_{q} U_{f}+U_{e m}\right)+c^{2} \boldsymbol{\nabla} \cdot\left(\sum_{q} \boldsymbol{G}_{f}+\boldsymbol{G}_{e m}\right) & =0
\end{aligned}
$$

where the electromagnetic field quantities are defined by ${ }^{29-31}$

$$
\begin{aligned}
& U_{e m}=\frac{1}{2}\left(\epsilon_{0} E^{2}+\frac{B^{2}}{\mu_{0}}\right)=\overline{\overline{\boldsymbol{T}}}_{e m}: \overline{\overline{\boldsymbol{I}}} \quad \text { field energy density, } \\
& \boldsymbol{G}_{e m}=\epsilon_{0}(\boldsymbol{E} \times \boldsymbol{B}) \quad \text { field momentum density, } \\
& \overline{\overline{\boldsymbol{T}}}_{e m}=\epsilon_{0}\left(\frac{E^{2}}{2} \overline{\overline{\boldsymbol{I}}}-\boldsymbol{E} \boldsymbol{E}\right)+\frac{1}{\mu_{0}}\left(\frac{B^{2}}{2} \overline{\overline{\boldsymbol{I}}}-\boldsymbol{B B}\right) \quad \text { field stress tensor. }
\end{aligned}
$$

The system of plasma fluid equations is given in the conservation form by

$$
\frac{\partial \rho}{\partial t}=-\nabla \cdot \boldsymbol{j} \quad \text { charge density }
$$

$\frac{\partial}{\partial t}\left(\sum_{q} \boldsymbol{G}_{f}+\boldsymbol{G}_{e m}\right)=-\boldsymbol{\nabla} \cdot\left(\sum_{q} \overline{\overline{\boldsymbol{T}}}_{f}+\overline{\overline{\boldsymbol{T}}}_{e m}\right)$ momentum density,

$$
\frac{\partial}{\partial t}\left(\sum_{q} U_{f}+U_{e m}\right)=-c^{2} \nabla \cdot\left(\sum_{q} \boldsymbol{G}_{f}+\boldsymbol{G}_{e m}\right) \quad \text { energy density. }
$$

Integration over a volume $V$ gives the integral form of the conservation equations

$$
\begin{gathered}
\int_{V} \dot{\rho} d^{3} r=-\oint_{S} \boldsymbol{j} \cdot d^{2} \boldsymbol{r} \text { charge } \\
\int_{V}\left(\sum_{q} \dot{\boldsymbol{G}}_{f}+\dot{\boldsymbol{G}}_{e m}\right) d^{3} r=-\oint_{S}\left(\sum_{q} \overline{\overline{\boldsymbol{T}}}_{f}+\overline{\overline{\boldsymbol{T}}}_{e m}\right) \cdot d^{2} \mathbf{r} \\
\int_{V}\left(\sum_{q} \dot{U}_{f}+\dot{U}_{e m}\right) d^{3} r=-c^{2} \oint_{S}\left(\sum_{q} \boldsymbol{G}_{f}+\boldsymbol{G}_{e m}\right) \cdot d^{2} \mathbf{r}
\end{gathered}
$$

Now, the laws of Ampère and Faraday give

$$
\begin{aligned}
& \boldsymbol{E} \cdot \nabla \times \boldsymbol{B}=\mu_{0} \sum_{q} \boldsymbol{j} \cdot \boldsymbol{E}+\frac{1}{c^{2}} \boldsymbol{E} \cdot \dot{\boldsymbol{E}}, \\
& \boldsymbol{B} \cdot \nabla \times \boldsymbol{E}=-\boldsymbol{B} \cdot \dot{\boldsymbol{B}} .
\end{aligned}
$$

The subtraction of these two equations gives

$$
\boldsymbol{\nabla} \cdot\left(\frac{\boldsymbol{E} \times \boldsymbol{B}}{\mu_{0}}\right)=-\sum_{q} \boldsymbol{j} \cdot \boldsymbol{E}-\frac{1}{2}\left(\epsilon_{0} \dot{E^{2}}+\frac{\dot{B^{2}}}{\mu_{0}}\right),
$$

hence,

$$
\boldsymbol{\nabla} \cdot \boldsymbol{S}_{e m}=-\sum_{q} \boldsymbol{j} \cdot \boldsymbol{E}-\dot{U}_{e m}
$$

where

$$
S_{e m}=\frac{\boldsymbol{E} \times \boldsymbol{B}}{\mu_{0}}=c^{2} \boldsymbol{G}_{e m}
$$

is the vector of Poynting. Integration over $V$ yields the theorem of Poynting,

$$
\int_{V}\left(\dot{U}_{e m}+\sum_{q} \boldsymbol{j} \cdot \boldsymbol{E}\right) d^{3} r=-\oint_{S} \mathbf{S}_{e m} \cdot d^{2} \mathrm{r}
$$

In general, the angular momentum density is defined by $\boldsymbol{L}=\boldsymbol{r} \times \boldsymbol{G}$. It can be shown that, due to both the conservation of momentum density 
and the symmetry of $\overline{\bar{T}}$, the angular momentum density is also conserved.

Finally, consider the quantity

$$
\begin{aligned}
\boldsymbol{\nabla} & \cdot\left[\boldsymbol{r} \cdot\left(\sum_{q} \overline{\overline{\boldsymbol{T}}}_{f}+\overline{\overline{\boldsymbol{T}}}_{e m}\right)\right] \\
& =\boldsymbol{\nabla} \boldsymbol{r}:\left(\sum_{q} \overline{\overline{\boldsymbol{T}}}_{f}+\overline{\overline{\boldsymbol{T}}}_{e m}\right)+\boldsymbol{r} \cdot \boldsymbol{\nabla} \cdot\left(\sum_{q} \overline{\overline{\boldsymbol{T}}}_{f}+\overline{\overline{\boldsymbol{T}}}_{e m}\right)^{T} \\
& =\overline{\overline{\boldsymbol{I}}}:\left(\sum_{q} \overline{\overline{\boldsymbol{T}}}_{f}+\overline{\overline{\boldsymbol{T}}}_{e m}\right)+\boldsymbol{r} \cdot \boldsymbol{\nabla} \cdot\left(\sum_{q} \overline{\overline{\boldsymbol{T}}}_{f}+\overline{\overline{\boldsymbol{T}}}_{e m}\right) \\
& =\overline{\overline{\boldsymbol{I}}}:\left(\sum_{q} \overline{\overline{\boldsymbol{T}}}_{f}+\overline{\overline{\boldsymbol{T}}}_{e m}\right)-\boldsymbol{r} \cdot\left(\sum_{q} \dot{\boldsymbol{G}}_{f}+\dot{\boldsymbol{G}}_{e m}\right) .
\end{aligned}
$$

Integration over $V$ yields

$$
\begin{aligned}
\oint_{S}[\boldsymbol{r} & \left.\cdot\left(\sum_{q} \overline{\overline{\boldsymbol{T}}}_{f}+\overline{\boldsymbol{T}}_{e m}\right)\right] \cdot d^{2} \boldsymbol{r} \\
= & \int_{V} \overline{\overline{\boldsymbol{I}}}:\left(\sum_{q} \overline{\overline{\boldsymbol{T}}}_{f}+\overline{\overline{\boldsymbol{T}}}_{e m}\right) d^{3} r \\
& -\int_{V} r \cdot\left(\sum_{q} \dot{\boldsymbol{G}}_{f}+\cdot \boldsymbol{G}_{e m}\right) d^{3} r .
\end{aligned}
$$

Assuming that all quantities are bounded, one takes the time average over a long period of time according to the definition

$$
\langle\dot{f}\rangle=\frac{1}{t} \int_{0}^{t} \dot{f} d t=\frac{f(t)-f(0)}{t} \underset{t \rightarrow \infty}{\rightarrow} 0 .
$$

Hence,

$$
\int_{V}\left\langle\overline{\overline{\boldsymbol{I}}}:\left(\sum_{q} \overline{\overline{\boldsymbol{T}}}_{f}+\overline{\overline{\boldsymbol{T}}}_{e m}\right)\right\rangle d^{3} r=\oint_{S}\left\langle\boldsymbol{r} \cdot\left(\sum_{q} \overline{\overline{\boldsymbol{T}}}_{f}+\overline{\overline{\boldsymbol{T}}}_{e m}\right)\right\rangle \cdot d^{2} \boldsymbol{r} .
$$

This is a general form of the virial theorem. ${ }^{32}$ Substituting the expressions for the stress dyadics

$$
\begin{aligned}
\int_{V}\left\langle\sum_{q}\left[\gamma n m v^{2}+\left(3+\frac{\gamma_{A}}{\gamma_{A}-1} \gamma^{2} \beta^{2}\right) p\right]+U_{e m}\right\rangle d^{3} r \\
=\oint_{S}\left\langle\boldsymbol{r} \cdot \sum_{q}\left[\gamma n m \boldsymbol{v} \boldsymbol{v}+\left(\overline{\overline{\boldsymbol{I}}}+\frac{\gamma_{A}}{\gamma_{A}-1} \gamma^{2} \frac{\boldsymbol{v} \boldsymbol{v}}{c^{2}}\right) p\right]\right\rangle \cdot d^{2} r \\
-\oint_{S}\left\langle\boldsymbol{r} \cdot\left(\epsilon_{0} \boldsymbol{E} \boldsymbol{E}+\frac{\boldsymbol{B} \boldsymbol{B}}{\mu_{0}}\right)+\boldsymbol{r} U_{e m}\right\rangle \cdot d^{2} r .
\end{aligned}
$$

Since, at the edge of the plasma configuration, the mass density $\mathrm{nm}$ and the pressure $p$ vanish for all species (mass discontinuities are ignored), the virial theorem shows that the configuration is contained by the field surface terms. The field energy density existing beyond the plasma surface represents a back pressure acting on the plasma fluid. This shows that a plasma fluid cannot be self-contained, since all terms in the volume integral are positive.

\section{BOUNDARY CONDITIONS AND ENERGY DENSITY DISTRIBUTION}

The plasma fluid and electromagnetic field variables must satisfy several boundary conditions at the fluid-vacuum interface, as follows. Let $\hat{\boldsymbol{n}}$ denotes the unit vector normal to the fluid-vacuum interface and $\langle\langle X\rangle\rangle$ the increment of any quantity $X$ across the boundary in the direction $\hat{\boldsymbol{n}}$. For the interface between two fluids,

$$
\hat{\boldsymbol{n}} \cdot\langle\langle\boldsymbol{v}\rangle\rangle=0 \text {. }
$$

For the fluid-vacuum interface,

$$
\hat{\boldsymbol{n}} \cdot \boldsymbol{v}=0 .
$$

Gauss's law in the fluid-vacuum interface gives

$$
\begin{gathered}
\nabla \cdot \boldsymbol{E}=\frac{1}{\epsilon_{0}} \sum_{q} \rho \Rightarrow \hat{\boldsymbol{n}} \cdot\langle\langle\boldsymbol{E}\rangle\rangle=\frac{1}{\epsilon_{0}} \sum_{q} \sigma \\
\nabla \cdot \boldsymbol{B}=0 \Rightarrow \hat{\boldsymbol{n}} \cdot\langle\langle\boldsymbol{B}\rangle\rangle=0,
\end{gathered}
$$

where $\sigma$ designates the surface charge density associated with species q. Also, the laws of Faraday and Ampère give

$$
\begin{gathered}
\boldsymbol{\nabla} \times \boldsymbol{E}=-\frac{\partial \boldsymbol{B}}{\partial t} \Rightarrow \hat{\boldsymbol{n}} \times\langle\langle\boldsymbol{E}\rangle\rangle=0 \\
\nabla \times \boldsymbol{B}=\mu_{0} \sum_{q} \rho \boldsymbol{v}+\frac{1}{c^{2}} \frac{\partial \boldsymbol{E}}{\partial t} \Rightarrow \hat{\boldsymbol{n}} \times\langle\langle\boldsymbol{B}\rangle\rangle=\mu_{0} \sum_{q} \boldsymbol{K},
\end{gathered}
$$

where $\boldsymbol{K}$ designates the surface current density.

The equation of momentum conservation for species $q$ is given by

$$
n m \frac{d}{d t}\left[\gamma\left(1+\frac{\gamma_{A}}{\gamma_{A}-1} \frac{\gamma p}{n m c^{2}}\right) \boldsymbol{v}\right]=-\nabla p+n q(\boldsymbol{E}+\boldsymbol{v} \times \boldsymbol{B}) .
$$

Thus, using the laws of Gauss, Ampère, and Faraday,

$$
\begin{aligned}
n m & \frac{d}{d t}\left[\gamma\left(1+\frac{\gamma_{A}}{\gamma_{A}-1} \frac{\gamma p}{n m c^{2}}\right) \boldsymbol{v}\right] \\
= & -\nabla p+\epsilon_{0}[\nabla \cdot(\boldsymbol{E} \boldsymbol{E})-(\boldsymbol{E} \cdot \boldsymbol{\nabla}) \boldsymbol{E}] \\
& -\epsilon_{0} \frac{\partial}{\partial t}(\boldsymbol{E} \times \boldsymbol{B})-\left[\epsilon_{0} \boldsymbol{E} \times(\boldsymbol{\nabla} \times \boldsymbol{E})+\frac{1}{\mu_{0}} \boldsymbol{B} \times(\boldsymbol{\nabla} \times \boldsymbol{B})\right] .
\end{aligned}
$$

Using the following relations,

$$
\begin{aligned}
(\boldsymbol{\nabla} \cdot \boldsymbol{E}) \boldsymbol{E} & =\boldsymbol{\nabla} \cdot(\boldsymbol{E} \boldsymbol{E})-(\boldsymbol{E} \cdot \boldsymbol{\nabla}) \boldsymbol{E} \\
& =(\boldsymbol{\nabla} \boldsymbol{E}) \cdot \boldsymbol{E}-(\boldsymbol{E} \times \boldsymbol{\nabla}) \times \boldsymbol{E}, \\
(\boldsymbol{\nabla} \cdot \boldsymbol{B}) \boldsymbol{B} & =\boldsymbol{\nabla} \cdot(\boldsymbol{B} \boldsymbol{B})-(\boldsymbol{B} \cdot \boldsymbol{\nabla}) \boldsymbol{B}=0, \\
\boldsymbol{E} \times(\boldsymbol{\nabla} \times \boldsymbol{E}) & =(\boldsymbol{\nabla} \boldsymbol{E}) \cdot \boldsymbol{E}-(\boldsymbol{E} \cdot \boldsymbol{\nabla}) \boldsymbol{E}=\frac{\boldsymbol{\nabla} E^{2}}{2}-(\boldsymbol{E} \cdot \boldsymbol{\nabla}) \boldsymbol{E}, \\
\boldsymbol{B} \times(\boldsymbol{\nabla} \times \boldsymbol{B}) & =(\boldsymbol{\nabla} \boldsymbol{B}) \cdot \boldsymbol{B}-(\boldsymbol{B} \cdot \boldsymbol{\nabla}) \boldsymbol{B}=\frac{\boldsymbol{\nabla} B^{2}}{2}-(\boldsymbol{B} \cdot \boldsymbol{\nabla}) \boldsymbol{B},
\end{aligned}
$$

the equation of momentum conservation becomes

$$
\begin{aligned}
n m & \frac{d}{d t}\left[\gamma\left(1+\frac{\gamma_{A}}{\gamma_{A}-1} \frac{\gamma p}{n m c^{2}}\right) \boldsymbol{v}\right] \\
& =-\epsilon_{0} \frac{\partial}{\partial t}(\boldsymbol{E} \times \boldsymbol{B})-\boldsymbol{\nabla} \cdot\left[p \overline{\overline{\boldsymbol{I}}}+\frac{1}{2}\left(\epsilon_{0} E^{2}+\frac{B^{2}}{\mu_{0}}\right) \overline{\overline{\boldsymbol{I}}}-\left(\epsilon_{0} \boldsymbol{E} \boldsymbol{E}+\frac{\boldsymbol{B} \boldsymbol{B}}{\mu_{0}}\right)\right] .
\end{aligned}
$$


Furthermore,

$$
\begin{aligned}
n m \frac{d}{d t}\left[\gamma\left(1+\frac{\gamma_{A}}{\gamma_{A}-1} \frac{\gamma p}{n m c^{2}}\right) \boldsymbol{v}\right]= & n m \frac{\partial}{\partial t}\left[\gamma\left(1+\frac{\gamma_{A}}{\gamma_{A}-1} \frac{\gamma p}{n m c^{2}}\right) \boldsymbol{v}\right] \\
& +n m \gamma\left(1+\frac{\gamma_{A}}{\gamma_{A}-1} \frac{\gamma p}{n m c^{2}}\right) \boldsymbol{v} \cdot \nabla \boldsymbol{v} \\
& +n m v^{2} \nabla\left[\gamma\left(1+\frac{\gamma_{A}}{\gamma_{A}-1} \frac{\gamma p}{n m c^{2}}\right)\right]
\end{aligned}
$$

and

$$
\begin{aligned}
\boldsymbol{\nabla} \cdot\left(\epsilon_{0} \boldsymbol{E} \boldsymbol{E}+\frac{1}{\mu_{0}} \boldsymbol{B} \boldsymbol{B}\right)= & \epsilon_{0}(\boldsymbol{E} \cdot \boldsymbol{\nabla}) \boldsymbol{E}+\frac{1}{\mu_{0}}(\boldsymbol{B} \cdot \boldsymbol{\nabla}) \boldsymbol{B}+\left(\sum_{q} \rho\right) \boldsymbol{E} \\
= & \epsilon_{0}(\boldsymbol{E} \cdot \boldsymbol{\nabla}) \boldsymbol{E}+\frac{1}{\mu_{0}}(\boldsymbol{B} \cdot \boldsymbol{\nabla}) \boldsymbol{B} \\
& -\sum_{q} \rho\left(\boldsymbol{\nabla} \phi+\frac{\partial \boldsymbol{A}}{\partial t}\right) .
\end{aligned}
$$

Denoting an infinitesimal displacement either inside the fluid or from one side of the fluid boundary to the other by $\delta \boldsymbol{r}$, the equation of momentum conservation gives

$$
\begin{aligned}
n m \frac{\partial}{\partial t} & {\left[\gamma\left(1+\frac{\gamma_{A}}{\gamma_{A}-1} \frac{\gamma p}{n m c^{2}}\right) \boldsymbol{v} \cdot \delta \boldsymbol{r}\right] } \\
& +n m \gamma\left(1+\frac{\gamma_{A}}{\gamma_{A}-1} \frac{\gamma p}{n m c^{2}}\right)(\boldsymbol{v} \cdot \nabla \boldsymbol{v}) \cdot \delta \boldsymbol{r} \\
& +n m v^{2} \delta\left[\gamma\left(1+\frac{\gamma_{A}}{\gamma_{A}-1} \frac{\gamma p}{n m c^{2}}\right)\right] \\
= & -\epsilon_{0} \delta \boldsymbol{r} \cdot \frac{\partial}{\partial t}(\boldsymbol{E} \times \boldsymbol{B})-\delta\left[p+\frac{1}{2}\left(\epsilon_{0} E^{2}+\frac{B^{2}}{\mu_{0}}\right)\right] \\
& +\left[\epsilon_{0}(\boldsymbol{E} \cdot \boldsymbol{\nabla}) \boldsymbol{E}+\frac{1}{\mu_{0}}(\boldsymbol{B} \cdot \boldsymbol{\nabla}) \boldsymbol{B}\right] \cdot \delta \boldsymbol{r} \\
& -\sum_{q} \rho \delta \phi-\sum_{q} \rho \delta \boldsymbol{r} \cdot \frac{\partial \boldsymbol{A}}{\partial t} .
\end{aligned}
$$

Taking the limit $\delta \boldsymbol{r} \rightarrow 0$,

$$
\begin{aligned}
& n m v^{2} \delta\left[\gamma\left(1+\frac{\gamma_{A}}{\gamma_{A}-1} \frac{\gamma p}{n m c^{2}}\right)\right] \\
& \quad+\delta\left(p+\frac{1}{2}\left(\epsilon_{0} E^{2}+\frac{B^{2}}{\mu_{0}}\right)\right)+\sum_{q} \rho \delta \phi=0 .
\end{aligned}
$$

Hence,

$$
\left\langle\left\langle n m \gamma\left(1+\frac{\gamma_{A}}{\gamma_{A}-1} \frac{\gamma p}{n m c^{2}}\right) v^{2}+p+\sum_{q} \rho \phi+U_{e m}\right\rangle\right\rangle=0 .
$$

This indicates that the sum of the kinetic energy density, of the pressure of each plasma species, and of the total electrostatic forces is balanced across each interface by changes in the field energy density. For a quasi-neutral plasma with negligible kinetic energy, this simply corresponds to magnetic confinement,

$$
\left\langle\left\langle p+\frac{B^{2}}{2 \mu_{0}}\right\rangle\right\rangle=0 \text {. }
$$

\section{COMMENTS AND CONCLUSIONS}

A consistent set of hydrodynamic and Maxwell equations was obtained applying Hamilton's principle to a perfect fully relativistic plasma fluid. This derivation of plasma dynamics from general principles shows the strength and validity of fluid descriptions. The connection between the Eulerian and Lagrangian formulations for the variational principle was demonstrated by means of the Clebsch representation for the canonical momentum of each fluid element, which is implicit in the Eulerian formulation. The Euler potentials (also known as Clebsch or Monge potentials) in the Clebsch representation are associated with constraints in the variational principle. It was shown that each Euler potential or Lagrangian multiplier is governed by an equation of motion which defines the evolution of the canonical momentum from its initial distribution. In this sense, the Clebsch representation is equivalent to the Lagrangian picture. However, it is possible to eliminate the Lagrangian multipliers in such a way that the final equation of motion for the canonical momentum depends only on the mean field values, corresponding exactly to the Eulerian formulation of fluid flow.

It was shown that the Lin coefficient for fluid flow corresponds to the reference (initial) distribution of canonical momentum for each charged fluid element. Furthermore, the Lagrangian multiplier associated with the continuity constraint for fluid flow corresponds to the longitudinal Euler potential in the Clebsch representation. A general discussion about helicity injection in the plasma system was carried out. In particular, a generalized form of Woltjer's theorem for the total helicity was obtained by a simple application of the transport theorem. The helicity changes are definitively associated with current injection in tokamaks and with the relaxation of toroidal plasmas toward an equilibrium state, and one may envisage a link with the excitation of turbulence in small (layer or pointlike) regions in the fluid. In this case, the equation of motion for the longitudinal potential must lead to a discontinuous solution, such as a shock wave. But this poses a challenge for further studies.

A canonical vorticity vector was defined in terms of the curl of the canonical momentum. It was shown that the fully relativistic canonical vorticity density is a Cauchy invariant, partially integrating the equations of motion of the plasma fluid. This also shows that a magnetic field can introduce flow vorticity in an inviscid fluid, with important implications in mass accretion and magnetic dynamo-effects.

It was shown that the Lagrangian formulation leads directly to an energy integral including the plasma fluid and the electromagnetic fields. Following standard procedures, the conservation equations including plasma fluid and electromagnetic field interactions were written in an integral form. In particular, a general form of the virial theorem was obtained. A general expression for the balance between plasma fluid and electromagnetic field forces was also obtained by the introduction of an infinitesimal displacement either inside the fluid or across one side of the fluid boundary to the other.

Finally, it must be pointed out that all the results presented in this paper can be applied to an extended version of the gravitoelectromagnetic theory. Gravitoelectromagnetic field equations give a result 
similar to the electromagnetic field theory with the following analogies:

$$
\begin{aligned}
& \boldsymbol{E} \rightarrow \boldsymbol{E}_{g}, \quad \boldsymbol{B} \rightarrow \boldsymbol{B}_{g}, \\
& \phi \rightarrow \phi_{g}, \quad \boldsymbol{A} \rightarrow \boldsymbol{A}_{g}, \\
& \epsilon_{0} \rightarrow-\frac{1}{4 \pi G}, \quad \mu_{0} \rightarrow-\frac{4 \pi G}{c^{2}},
\end{aligned}
$$

where $\boldsymbol{E}_{g}$ and $\boldsymbol{B}_{g}$ are the gravitoelectromagnetic fields, $\phi_{g}$ and $\boldsymbol{A}_{g}$ are the corresponding potentials $\left(\phi_{g}\right.$, in particular, is the Newtonian potential), $G$ is the gravitational constant, and the charge $q$ is replaced by the mass $m$ in a single species fluid. Using this simple analogies, the dynamic equations which describe the evolution of a continuous mass distribution are readily obtained, according to the same variational formulation. These equations constitute an extended version of the gravitomagnetic theory originally proposed by Thirring in $1918 .{ }^{33-36}$ The first application of this weak-field approach to Einstein's general relativity theory resulted in the astronomical calculations performed by Lense and Thirring ${ }^{37}$ and reviewed by Pfister ${ }^{38}$ of the forces on a test mass due to the rotation of a large central mass. This so-called Lense-Thirring relativistic effect has attracted great interest in recent years as it became barely possible to detect the effect using terrestrial spacecrafts. ${ }^{39}$ Actually, the effect was recently confirmed at an accuracy of $19 \%$ by measurements taken by the Gravity Probe B space experiment. ${ }^{40}$ The extended version of gravitoelectromagnetism can be used to describe, for example, the dynamics of a galactic system formed by a very large number of stars plus the surrounding gas. This application is the subject of work currently in progress.

\section{ACKNOWLEDGMENTS}

This work was supported by a grant provided by the Programa de Capacitação Institucional: Diretoria de Pesquisa e Desenvolvimento/Comissão Nacional de Energia Nuclear (CNEN).

\section{APPENDIX A: COVARIANT FORMULATION}

\section{Preliminary remarks}

The contravariant coordinates' four-vector is

$$
x^{\mu}=(c t, \boldsymbol{r}),
$$

with the corresponding covariant form

$$
x_{\mu}=\eta_{\mu \nu} x^{\nu}=(-c t, \boldsymbol{r}),
$$

where

$$
\eta_{\mu \nu}=\left(\begin{array}{cc}
-1 & \mathbf{0} \\
\mathbf{0} & \overline{\overline{\mathbf{I}}}
\end{array}\right)=\eta^{\mu \nu}
$$

is the Minkowsky (flat-space metric) tensor. Note that

$$
\eta^{\mu \rho} \eta_{\nu \rho}=\left(\begin{array}{cc}
1 & \mathbf{0} \\
\mathbf{0} & \overline{\overline{\mathbf{I}}}
\end{array}\right)=\delta_{\nu}^{\mu}
$$

is the Kronecker mixed tensor. Also,

$$
\eta^{\mu \nu} \eta_{\mu \nu}=4
$$

The covariant gradient four-vector is

$$
\partial_{\mu} \equiv \frac{\partial}{\partial x^{\mu}} \equiv\left(\frac{1}{c} \frac{\partial}{\partial t}, \nabla\right),
$$

with the corresponding contravariant form

$$
\partial^{\mu} \equiv \eta^{\mu \nu} \partial_{\nu} \equiv\left(-\frac{1}{c} \frac{\partial}{\partial t}, \nabla\right) .
$$

In general, the invariant scalar product of two four-vectors is

$$
a_{\mu} b^{\mu}=a_{0} b^{0}+\boldsymbol{a} \cdot \boldsymbol{b}=-a_{0} b_{0}+\boldsymbol{a} \cdot \boldsymbol{b} .
$$

In particular,

$$
x^{2}=x_{\mu} x^{\mu}=-c^{2} t^{2}+r^{2} .
$$

The scalar product of $\partial_{\mu}$ with itself gives the d'Alembertian operator,

$$
\square^{2} \equiv \partial_{\mu} \partial^{\mu} \equiv \eta^{\mu \nu} \frac{\partial}{\partial x^{\mu}} \frac{\partial}{\partial x^{\nu}} \equiv-\frac{1}{c^{2}} \frac{\partial^{2}}{\partial t^{2}}+\nabla^{2},
$$

which is also invariant. The proper time interval $d \tau$ is defined by

$$
c^{2} d \tau^{2}=-\eta_{\mu \nu} d x^{\mu} d x^{\nu}=c^{2} d t^{2}-d \boldsymbol{r}^{2}=c^{2}\left(1-\frac{v^{2}}{c^{2}}\right) d t^{2},
$$

where

$$
\boldsymbol{v}=\frac{d \boldsymbol{r}}{d t}
$$

is the fluid velocity. The contravariant form of the fluid fourvelocity is

$$
v^{\mu}=\frac{d x^{\mu}}{d \tau}=\gamma(c, \boldsymbol{v})
$$

where

$$
\gamma=\frac{d t}{d \tau}=\left(1-\frac{v^{2}}{c^{2}}\right)^{-1 / 2}=\frac{1}{\sqrt{1-v^{2} / c^{2}}}
$$

is the Lorentz factor. The scalar product gives

$$
v_{\mu} v^{\mu}=\gamma(-c, \boldsymbol{v}) \cdot \gamma(c, \boldsymbol{v})=\gamma^{2}\left(-c^{2}+v^{2}\right)=-c^{2} .
$$

\section{Covariant equations of motion}

The Lagrangian can be written in a manifestly covariant form as follows:

$$
\mathcal{L}=\sum_{q}\left(-\stackrel{\circ}{U}+j_{\mu} A^{\mu}\right)-\frac{\Lambda}{4 \mu_{0}},
$$

where the proper energy density $\stackrel{\circ}{U}$, sum of the rest mass and thermal energy densities, is given by

$$
\stackrel{\circ}{U}=\stackrel{\circ}{n} m c^{2}+\frac{p}{\gamma_{A}-1} .
$$

The number density in the rest frame, which is denoted by the upper circle label, is related to the number density in the moving 
frame by the Lorentz factor $\stackrel{\circ}{n}=n / \gamma$. The pressure $p$ is an invariant under a proper Lorentz transformation, and $\gamma_{A}$ is a function of the rest frame temperature $T=\gamma T$. The term $j_{\mu} A^{\mu}$ corresponds to the interaction energy between the fluid and the electromagnetic field,

$$
j_{\mu} A^{\mu}=(-\rho c, \boldsymbol{j}) \cdot\left(\frac{\phi}{c}, \boldsymbol{A}\right)=-\rho \phi+\boldsymbol{j} \cdot \boldsymbol{A} .
$$

The electromagnetic field tensor is given by ${ }^{10}$

$$
F^{\mu \nu}=\left(\begin{array}{cc}
0 & \boldsymbol{E} / c \\
-\boldsymbol{E} / c & \overline{\overline{\boldsymbol{\epsilon}}} \cdot \boldsymbol{B}
\end{array}\right)
$$

and

$$
F_{\mu \nu}=\eta_{\mu \rho} F^{\rho \sigma} \eta_{\sigma \nu}=\left(\begin{array}{cc}
0 & -\boldsymbol{E} / c \\
\boldsymbol{E} / c & \overline{\overline{\boldsymbol{\epsilon}}} \cdot \boldsymbol{B}
\end{array}\right),
$$

where $\overline{\bar{\epsilon}}$ is the totally antisymmetric Levi-Civita tensor in three dimensions and $\eta_{\mu \nu}$ is the Minkowsky tensor. The first electromagnetic field invariant $\Lambda$ is given in terms of $F^{\mu \nu}$ by

$$
\begin{aligned}
\Lambda & =F_{\mu \nu} F^{\mu \nu}=\left(\begin{array}{cc}
0 & -\boldsymbol{E} / c \\
\boldsymbol{E} / c & \overline{\overline{\boldsymbol{\epsilon}}} \cdot \boldsymbol{B}
\end{array}\right):\left(\begin{array}{cc}
0 & \boldsymbol{E} / c \\
-\boldsymbol{E} / c & \overline{\overline{\boldsymbol{\epsilon}}} \cdot \boldsymbol{B}
\end{array}\right)^{T} \\
& =-\frac{2\left(E^{2}-c^{2} B^{2}\right)}{c^{2}}
\end{aligned}
$$

The anti-symmetric electromagnetic field tensor can be derived from the four-vector potential as follows:

$$
F^{\mu \nu}=\partial^{\mu} A^{\nu}-\partial^{\nu} A^{\mu},
$$

that is,

$$
\begin{aligned}
\left(\begin{array}{cc}
0 & \boldsymbol{E} / c \\
-\boldsymbol{E} / c & \overline{\overline{\boldsymbol{\epsilon}}} \cdot \boldsymbol{B}
\end{array}\right) & =\left(\begin{array}{cc}
-\frac{1}{c} \frac{\partial}{\partial t} \\
\boldsymbol{\nabla}
\end{array}\right)\left(\begin{array}{cc}
\frac{\phi}{c} & \boldsymbol{A}
\end{array}\right)-\left[\left(\begin{array}{cc}
-\frac{1}{c} \frac{\partial}{\partial t} \\
\boldsymbol{\nabla}
\end{array}\right)\left(\begin{array}{cc}
\frac{\phi}{c} & \boldsymbol{A}
\end{array}\right)\right]^{T} \\
& =\left(\begin{array}{cc}
0 & -\frac{1}{c} \frac{\partial \boldsymbol{A}}{\partial t}-\frac{1}{c} \nabla \phi \\
\frac{1}{c} \nabla \phi+\frac{1}{c} \frac{\partial \boldsymbol{A}}{\partial t} & \nabla \boldsymbol{A}-(\nabla \boldsymbol{A})^{T}
\end{array}\right) .
\end{aligned}
$$

Hence,

$$
\begin{aligned}
& \boldsymbol{E}=-\nabla \phi-\frac{\partial \boldsymbol{A}}{\partial t}, \\
& \boldsymbol{B}=\boldsymbol{\nabla} \times \boldsymbol{A} .
\end{aligned}
$$

The relation $F^{\mu \nu}=\partial^{\mu} A^{\nu}-\partial^{\nu} A^{\mu}$ is a consequence of gauge invariance of the second kind. The quantities $A_{\mu}(x)$ and $A_{\mu}(x)$ $-\partial_{\mu} f(x)$ are physically indistinguishable, so that $A^{\mu}$ can be required to satisfy Lorenz's condition

$$
\partial_{\mu} A^{\mu}=0 \Rightarrow \frac{1}{c^{2}} \frac{\partial \phi}{\partial t}+\nabla \cdot \boldsymbol{A}=0 .
$$

The inhomogeneous field equations can be written in terms of the four-potential as

$$
\square^{2} A^{\mu}=-\mu_{0} \sum_{q} j^{\mu}\left\{\begin{array}{l}
\square^{2} \phi=-\frac{1}{\epsilon_{0}} \sum_{q} \rho \\
\square^{2} \boldsymbol{A}=-\mu_{0} \sum_{q} \boldsymbol{j} .
\end{array}\right.
$$

In the covariant form, the total energy-momentum tensor $T^{\mu \nu}$ is given by the sum of the plasma fluid $T_{f}^{\mu \nu}$ and electromagnetic field $T_{e m}^{\mu \nu}$ tensors defined in Sec. VI

$$
\begin{aligned}
T_{f}^{\mu \nu} & =\left(\begin{array}{cc}
U_{f} & c \boldsymbol{G}_{f} \\
c \boldsymbol{G}_{f} & \overline{\overline{\boldsymbol{T}}}_{f}
\end{array}\right), \\
T_{e m}^{\mu \nu} & =\left(\begin{array}{cc}
U_{e m} & c \boldsymbol{G}_{e m} \\
c \boldsymbol{G}_{e m} & \overline{\bar{T}}_{e m}
\end{array}\right) .
\end{aligned}
$$

Hence, the total momentum and energy conservation equations can be written in the covariant form as follows:

$$
\begin{aligned}
& \partial_{\nu} T^{\mu \nu}=\left(\frac{1}{c} \frac{\partial}{\partial t}, \boldsymbol{\nabla}\right) . \\
& \left(\begin{array}{cc}
\sum_{q} U_{f}+U_{e m} & \sum_{q} c \boldsymbol{G}_{f}+c \boldsymbol{G}_{e m} \\
\sum_{q} c \boldsymbol{G}_{f}+c \boldsymbol{G}_{e m} & \sum_{q} \overline{\overline{\boldsymbol{T}}}_{f}+\overline{\overline{\boldsymbol{T}}}_{e m}
\end{array}\right)=0 .
\end{aligned}
$$

This equation describes the exchange of energy between the plasma fluid species and the electromagnetic field. The energy-momentum tensor of the perfect fluid is given in the covariant form by

$$
\begin{aligned}
T_{f}^{\mu \nu} & =p \eta^{\mu \nu}+(\stackrel{\circ}{U}+p) \frac{v^{\mu} v^{\nu}}{c^{2}} \\
& =p\left(\begin{array}{cc}
-1 & \mathbf{0} \\
\mathbf{0} & \overline{\overline{\boldsymbol{I}}}
\end{array}\right)+(\stackrel{\circ}{U}+p) \gamma^{2}\left(\begin{array}{cc}
1 & \boldsymbol{v} / c \\
\boldsymbol{v} / c & \boldsymbol{v} \boldsymbol{v} / c^{2}
\end{array}\right) .
\end{aligned}
$$

In terms of the electromagnetic field tensor $F^{\mu \nu}$, the fluid energymomentum equation for each species $q$ can be written as

$$
\partial_{\nu} T_{f}^{\mu \nu}=j_{\nu} F^{\mu \nu}
$$

Thus,

$$
\begin{aligned}
& \left(\frac{1}{c} \frac{\partial}{\partial t}, \nabla\right) \cdot\left(\begin{array}{cc}
\gamma^{2}\left(\stackrel{\circ}{U}+p \beta^{2}\right) & (\stackrel{\circ}{U}+p) \gamma^{2} \boldsymbol{v} / c \\
(\stackrel{\circ}{U}+p) \gamma^{2} \boldsymbol{v} / c & p \overline{\overline{\boldsymbol{I}}}+(\stackrel{\circ}{U}+p) \gamma^{2} \boldsymbol{v} \boldsymbol{v} / c^{2}
\end{array}\right) \\
& =(-\rho c, \boldsymbol{j}) \cdot\left(\begin{array}{cc}
0 & \boldsymbol{E} / c \\
-\boldsymbol{E} / c & \overline{\overline{\boldsymbol{\epsilon}}} \cdot \boldsymbol{B}
\end{array}\right)^{T} .
\end{aligned}
$$

The energy and momentum conservation equations are given, respectively, by the temporal component [cf. Eq. (63)]

$$
\frac{1}{c} \frac{\partial}{\partial t}\left[\gamma^{2}\left(\stackrel{\circ}{U}+p \beta^{2}\right)\right]+\nabla \cdot\left[(\stackrel{\circ}{U}+p) \gamma^{2} \frac{\boldsymbol{v}}{c}\right]=\boldsymbol{j} \cdot \frac{\boldsymbol{E}}{c},
$$

and by the spatial components [cf. Eq. (49)] of the previous equation 


$$
\begin{aligned}
& \frac{1}{c} \frac{\partial}{\partial t}\left[(\stackrel{\circ}{U}+p) \gamma^{2} \frac{\boldsymbol{v}}{c}\right]+\nabla \cdot\left[(\stackrel{\circ}{U}+p) \gamma^{2} \frac{\boldsymbol{v} \boldsymbol{v}}{c^{2}}+p \overline{\overline{\boldsymbol{I}}}\right] \\
& \quad=\rho E+j \times \boldsymbol{B} .
\end{aligned}
$$

The full set of equations which describes the dynamics of the plasma in the covariant form includes the equations of continuity, momentum and energy conservation, and Maxwell source equations, which can be written, respectively, as

$$
\begin{aligned}
\partial_{\mu} j^{\mu} & =0, \quad \text { continuity, } \\
\partial_{\nu} T_{f}^{\mu \nu} & =j_{\nu} F^{\mu \nu} \quad \text { momentum-energy, } \\
\partial_{\nu} F^{\mu \nu} & =\mu_{0} \sum_{q} j^{\mu} \quad \text { Gauss-Ampere }
\end{aligned}
$$

where

$$
F^{\mu \nu}=\partial^{\mu} A^{\nu}-\partial^{\nu} A^{\mu}, \quad \partial_{\mu} A^{\mu}=0 .
$$

The above set corresponds to a total of $5 q+4$ equations (here, $q$ indicates the number of species) in the $6 q+4$ variables: $j^{\mu}=q n(c, v), A^{\mu}=(\phi / c, A), p$, and $T$. The set is closed by the $q$ equations of state $p=n k_{B} T$. The condition of entropy conservation is satisfied by the above covariant set of equations constrained by the second law of thermodynamics as will be presently demonstrated. Multiplication of the energy-momentum conservation equation by the four-velocity yields

$$
\begin{aligned}
& v_{\mu} \partial_{\nu} T_{f}^{\mu \nu}=v_{\mu} j_{\nu} F^{\mu \nu}=\stackrel{\circ}{n} q v_{\mu} v_{\nu} F^{\mu \nu} \\
& =\stackrel{\circ}{q} q \gamma^{2}(-c, \boldsymbol{v}) \cdot\left(\begin{array}{cc}
0 & \boldsymbol{E} / c \\
-\boldsymbol{E} / c & \overline{\overline{\boldsymbol{\epsilon}}} \cdot \boldsymbol{B}
\end{array}\right) \cdot(-c, \boldsymbol{v}) \\
& =\stackrel{n}{q} q \gamma^{2}(-c, \boldsymbol{v}) \cdot\left(\begin{array}{c}
\boldsymbol{v} \cdot \boldsymbol{E} / c \\
\boldsymbol{E}+\boldsymbol{v} \times \boldsymbol{B}
\end{array}\right)=0 .
\end{aligned}
$$

Hence, replacing $T_{f}^{\mu \nu}$ by its covariant form,

$$
\begin{aligned}
v_{\mu} \partial_{\nu} T_{f}^{\mu \nu}= & v_{\mu} \partial_{\nu}\left[p \eta^{\mu \nu}+(\stackrel{\circ}{U}+p) \frac{v^{\mu} v^{\nu}}{c^{2}}\right] \\
= & v_{\mu} \partial^{\mu} p-\partial_{\nu}\left[(\stackrel{\circ}{U}+p) v^{\nu}\right] \\
& +\frac{1}{2}(\stackrel{\circ}{U}+p) v^{\nu} \underbrace{\partial_{\nu}\left(\frac{v_{\mu} v^{\mu}}{c^{2}}\right)}_{=0}=0 .
\end{aligned}
$$

Using the equation of continuity,

$$
\partial_{\mu}\left(\stackrel{\circ}{n} v^{\mu}\right)=0
$$

the previous equation becomes

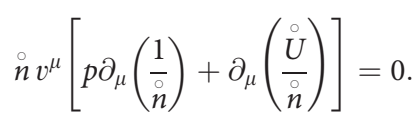

The second law of thermodynamics (cf. Appendix A 3) gives

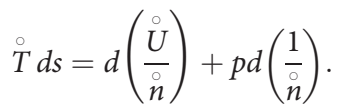

Thus,

$$
\stackrel{\circ}{n} \stackrel{\circ}{T}^{\mu} \partial_{\mu} s=p v^{\mu} \partial_{\mu} s=0,
$$

where $s$ is the invariant specific entropy of the species $q$ in the perfect fluid.

\section{Relativistic equation of state for a perfect fluid}

The fundamental thermodynamic relation (second law) in the rest frame, for either reversible or irreversible processes, reads

$$
\stackrel{\circ}{T} d\left(\begin{array}{c}
\circ \\
\frac{\sigma}{\circ} \\
n
\end{array}\right)=d\left(\begin{array}{c}
\stackrel{\vdots}{\circ} \\
\frac{\circ}{n}
\end{array}\right)+p d\left(\begin{array}{c}
1 \\
\vdots \\
n
\end{array}\right),
$$

where $\stackrel{\circ}{\sigma}$ is the entropy density in the local rest frame. The number density transforms from the rest frame to the frame moving with the fluid velocity $\boldsymbol{v}$ as $n=\gamma n$. The absolute temperature and the entropy density transform as $T=T / \gamma$ and $\stackrel{\circ}{\sigma}=\sigma / \gamma$, respectively. The specific entropy (proper entropy of a fluid element) is defined by

$$
s=\stackrel{\circ}{\sigma} / \stackrel{\circ}{n}=\sigma / n,
$$

and like $p$ is an invariant under a proper Lorentz transformation.

In general, the plasma fluid is characterized by the macroscopic variables $(n, \boldsymbol{v}, U, s, p, T)$, which evolve according to the continuity, momentum, and energy conservation equations making a total of five equations for eight variables. The temperature can be considered as defined by means of the fundamental relation. This relation shows that two of the thermodynamic scalar variables must be independent. Accordingly, three equations of state for the intensive variables $s, p$, and $T$ can be written in terms of the number and energy densities as independent variables in the rest frame

$$
s(\stackrel{\circ}{n}, \stackrel{\circ}{U}), \quad p(\stackrel{\circ}{n}, \stackrel{\circ}{U}), \quad \stackrel{\circ}{T}(\stackrel{\circ}{n}, \stackrel{\circ}{U}) .
$$

The fundamental relation can be written as

$$
\stackrel{\circ}{T} d s=\frac{1}{\stackrel{\circ}{\circ}} d \stackrel{\circ}{U}-\left(\frac{\stackrel{\circ}{U}+p}{\stackrel{\circ}{n}^{2}}\right) d \stackrel{\circ}{n} .
$$

Hence,

$$
\frac{\partial s}{\partial \stackrel{\circ}{U}}=\frac{1}{\stackrel{\circ}{\circ}}, \quad \frac{\partial s}{\partial n}=-\frac{\stackrel{\circ}{U}+p}{\stackrel{\circ}{n}^{2}} \stackrel{\circ}{T},
$$

and the integrability condition,

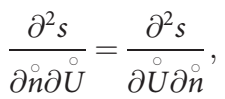

gives

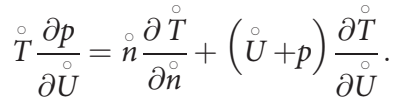

If the equation of state $T=T(\stackrel{\circ}{\circ}$,$) is given, the integrability con-$ dition can be used to obtain $p=p(\stackrel{\circ}{\mathrm{U}}, \stackrel{\circ}{\mathrm{U}})$ and the fundamental relation to obtain $s=s(\stackrel{\circ}{n}, \stackrel{\circ}{U})$. If any one of the three equations of state 
is given, the other two equations of state can be obtained. Therefore, taking into account the fundamental equation, only one scalar equation of state is needed to close the system of fluid equations. frame,

Using the definition of the plasma energy density in the rest

$$
\stackrel{\circ}{U}=\stackrel{\circ}{n} m c^{2}+\frac{p}{\gamma_{A}-1},
$$

the fundamental relation becomes

$$
\stackrel{\circ}{T} d s=d\left(\frac{\gamma_{A}}{\gamma_{A}-1} \frac{p}{\circ}\right)-\frac{d p}{\stackrel{\circ}{n}} .
$$

Assuming isentropic flow with $d s=0$, the fundamental relation gives either

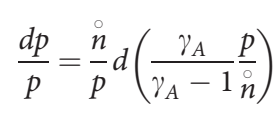

or the equivalent differential relation

$$
\frac{d p}{p}=\frac{\gamma_{A}^{2}}{\gamma_{A}-1} \frac{1}{\circ} d\left(\frac{\gamma_{A}-1}{\gamma_{A}} \stackrel{\circ}{\circ}\right) \text {. }
$$

As previously discussed, one equation of state relating $p, \stackrel{\circ}{n}$, and $T$ is needed to close the system. This equation, as well as the dependence of the coefficient $\gamma_{A}$ on the thermodynamic variables, comes from experimental results, phenomenological arguments, or kinetic theory. Assuming the ideal gas law in the rest frame $p=n k_{B} T$ as the given equation of state, the pressure is related to the temperature by

$$
\frac{d p}{p}=\frac{1}{\circ} d\left(\frac{\gamma_{A}}{\gamma_{A}-1} \stackrel{\circ}{T}\right) .
$$

Now, the "adiabatic" coefficient (or "polytropic index") of a relativistic perfect gas is ${ }^{27}$

$$
\gamma_{A}=\frac{1}{1-\frac{k_{B} \stackrel{\circ}{T}}{m c^{2}}\left[\frac{K_{3}\left(m c^{2} / k_{B} \stackrel{\circ}{T}\right)}{K_{2}\left(m c^{2} / k_{B} \stackrel{\circ}{T}\right)}-1\right]^{-1},}
$$

where $K_{\nu}$ are the modified Bessel functions of the second kind and order $\nu$. Hence,

$$
\begin{aligned}
\frac{d p}{p}= & \frac{m c^{2}}{k_{B} \stackrel{\circ}{T}} d\left(\frac{K_{3}\left(m c^{2} / k_{B} \stackrel{\circ}{T}\right)}{K_{2}\left(m c^{2} / k_{B} \stackrel{\circ}{T}\right)}\right) \\
= & d\left(\frac{m c^{2}}{k_{B} K_{3}\left(m c^{2} / k_{B} \stackrel{\circ}{T}\right)}\right) \\
& +\frac{d K_{2}\left(m c^{2} / k_{B} \stackrel{\circ}{T}\right)}{K_{2}\left(m c^{2} / k_{B} \stackrel{\circ}{T}\right)}-2 \frac{d\left(m c^{2} / k_{B} \stackrel{\circ}{T}\right)}{m c^{2} / k_{B} \stackrel{\circ}{T}},
\end{aligned}
$$

and the equation of state becomes ${ }^{27,41-43}$

$$
p \propto \frac{K_{2}\left(m c^{2} / k_{B} T\right)}{\left(m c^{2} / k_{B} \stackrel{\circ}{T}\right)^{2}} \exp \left(\frac{m c^{2}}{k_{B} \stackrel{\circ}{T} K_{3}\left(m c^{2} / k_{B}{ }^{2} / k_{B} \stackrel{\circ}{T}\right)}\right) .
$$

In the non-relativistic limit, the above equation of state reduces to the usual form of the adiabatic law for a monatomic gas,

$$
p \propto\left(k_{B} \stackrel{\circ}{T}\right)^{5 / 2} \text { for } k_{B} \stackrel{\circ}{T} \ll m c^{2},
$$

and in the extreme relativistic limit to [the threshold for pair production is $k_{B} T=2 m c^{2}$, with $\gamma_{A} \cong 1.3832$ and $\gamma_{A} /\left(\gamma_{A}-1\right)$ $\cong 3.6097]$

$$
p \propto\left(k_{B} \stackrel{\circ}{T}\right)^{4} \text { for } k_{B} \stackrel{\circ}{T} \gg m c^{2}
$$

If the relatively weak dependence of $\gamma_{A}$ on the temperature is neglected, the polytropic equation of state is obtained,

$$
p \propto\left(\frac{k_{B} T}{m c^{2}}\right)^{\gamma_{A} /\left(\gamma_{A}-1\right)} \sim \stackrel{\circ \gamma_{A}}{n} .
$$

Figures 1 and 2 show the variation with the temperature of the coefficient $\gamma_{A}$ and of the normalized pressure for a relativistic electron gas, respectively.

Note that the fundamental relation in a moving frame is

$$
\gamma T d s=d\left(\frac{p}{\gamma_{A}-1} \frac{\gamma}{n}\right)+p d\left(\frac{\gamma}{n}\right)
$$

Introducing the ideal gas law $p=n k_{B} T$,

$$
\frac{1}{k_{B}} d s=\frac{1}{k_{B} T} d\left(\frac{k_{B} T}{\gamma_{A}-1}\right)+\frac{\gamma_{A}}{\gamma_{A}-1} \frac{d \gamma}{\gamma}-\frac{d n}{n} .
$$

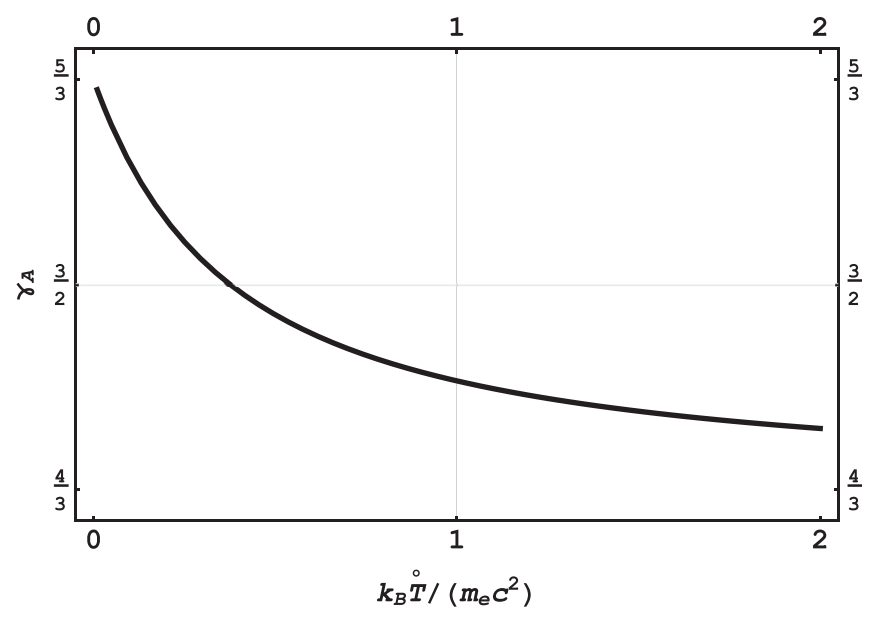

FIG. 1. Coefficient $\gamma_{A}$ for a relativistic electron gas. 


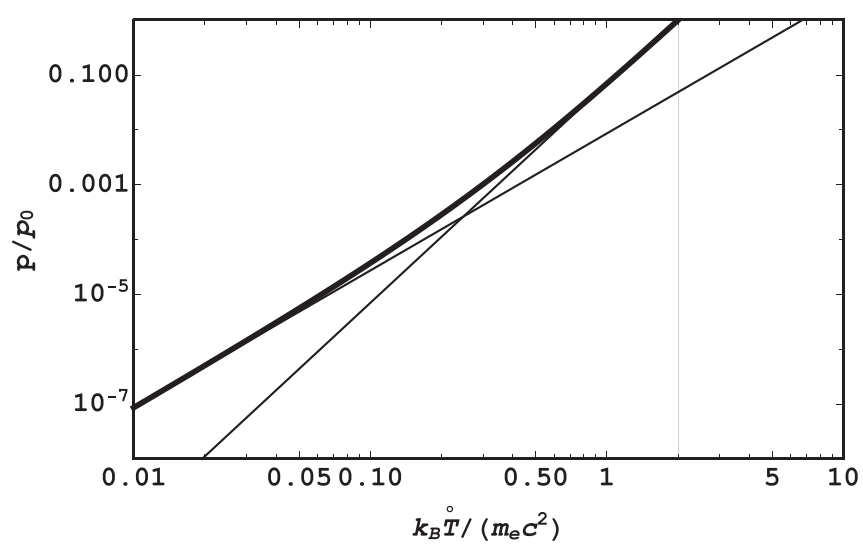

FIG. 2. Equation of state for a relativistic electron gas. The thin lines correspond to the asymptotic limits $p \propto\left(k_{B} \stackrel{\circ}{T}\right)^{5 / 2}$ and $p \propto\left(k_{B} \stackrel{\circ}{T}\right)^{4}$.

\section{APPENDIX B: KINEMATICAL CONCEPTS OF FLUID MOTION ${ }^{2}$}

\section{Kinematics of continuous systems}

Fluid flow is a continuous transformation of configuration space into itself (the Lagrangian map), parameterized by time $t$,

$$
\begin{array}{ccc}
\boldsymbol{r}_{0} & \boldsymbol{r} \\
\text { Initial position of a } & \rightarrow & \text { Position } \\
\text { fluid element or point } P & \text { at time } t .
\end{array}
$$

For fixed $\boldsymbol{r}_{0}$ and changing $t$, the transformation $\boldsymbol{r}=\boldsymbol{r}\left(\boldsymbol{r}_{0}, t\right)$ specifies the trajectory of $P$. For fixed $t$, it determines the transformation of $P$ from the initial position to the position at time $t$. The inverse transformation is denoted by $\boldsymbol{r}_{0}=\boldsymbol{r}_{0}(\boldsymbol{r}, t)$.

Eulerian or spatial variables $(\boldsymbol{r}, t)$ describe the evolution of a field function $f=f(\boldsymbol{r}, t)$, which corresponds to the value of $f$ experienced by a particle instantaneously at the position $r$. Lagrangian or material variables $\left(\boldsymbol{r}_{0}, t\right)$ single out individual points, so that $f=f\left(\boldsymbol{r}_{0}, t\right)$ is the value of $f$ experienced at time $t$ by the particle initially at $\boldsymbol{r}_{0}$. The convective derivative of $f$ is $d f / d t=\partial f\left(\boldsymbol{r}_{0}, t\right) / \partial t$ which gives the rate of change of $f$ following $P$, while $\partial f / \partial t$ $=\partial f(\boldsymbol{r}, t) / \partial t$ gives the rate of change of $f$ at a position $\boldsymbol{r}$.

Now, the velocity of a point $P$ is defined as a function of the material variables by

$$
\boldsymbol{v}=\frac{d \boldsymbol{r}\left(\boldsymbol{r}_{0}, t\right)}{d t}
$$

the functions $\boldsymbol{v}(\boldsymbol{r}, t)$ and $\boldsymbol{v}\left(\boldsymbol{r}_{0}, t\right)$ being related by the change of variables $\boldsymbol{r}=\boldsymbol{r}\left(\boldsymbol{r}_{0}, t\right)$ and $\boldsymbol{r}_{0}=\boldsymbol{r}_{0}(\boldsymbol{r}, t)$. Acceleration of the moving point is defined by

$$
\boldsymbol{a}=\frac{d \boldsymbol{v}(\boldsymbol{r}, t)}{d t}=\frac{\partial \boldsymbol{v}}{\partial t}+\boldsymbol{v} \cdot \boldsymbol{\nabla} \boldsymbol{v}
$$

The relation between convective and spatial derivatives is

$$
\frac{d f}{d t}=\frac{\partial f}{\partial t}+\boldsymbol{v} \cdot \nabla f
$$

which gives the rate of change of a quantity moving instantaneously with the velocity $\boldsymbol{v}$.

The Jacobian of the transformation $\boldsymbol{r}=\boldsymbol{r}\left(\boldsymbol{r}_{0}, t\right)$ is

$$
J=\left|\frac{\partial \boldsymbol{r}}{\partial \boldsymbol{r}_{0}}\right|=\left|\nabla_{0} \boldsymbol{r}\right|
$$

representing the dilatation of an infinitesimal volume as it follows the motion

$$
d^{3} r=J d^{3} r_{0}
$$

The transformation $\boldsymbol{r}_{0} \rightleftarrows \boldsymbol{r}$ is one-to-one, provided $J$ satisfies

$$
0<J<\infty \text {. }
$$

The Jacobian determinant can be written in the following form:

$$
J=\frac{1}{3} \overline{\bar{C}}^{T}: \nabla_{0} \boldsymbol{r}
$$

where $\overline{\bar{C}}$ is the dyadic of the cofactors of the elements of $\nabla_{0} r$.

The inverse of $\nabla_{0} r$ is

$$
\begin{aligned}
\frac{d J}{d t} & =\left(\overline{\bar{C}}^{T} \cdot \nabla_{0} \boldsymbol{r}\right): \boldsymbol{\nabla} \boldsymbol{v} \\
& =\left|\nabla_{0} \boldsymbol{r}\right| \overline{\overline{\mathbf{I}}}: \nabla \boldsymbol{v}=J \boldsymbol{\nabla} \cdot \boldsymbol{v} .
\end{aligned}
$$

This expression is the statement of Euler's theorem, namely,

$$
\frac{d J}{d t}=J \nabla \cdot \boldsymbol{v}
$$

For an incompressible fluid, $\boldsymbol{\nabla} \cdot \boldsymbol{v}=0$ and $d J / d t=0$.

\section{The transport theorem}

Consider the integral

$$
\int_{V} f(\boldsymbol{r}, t) d^{3} r
$$

performed over a piece of fluid occupying a volume $V=V(\boldsymbol{r}, t)$ at time $t$ and a volume $V_{0}=V(0)$ when $t=0$. The volume $V$ is at all times composed of the same particles. Therefore, by changing coordinates $\boldsymbol{r} \rightarrow \boldsymbol{r}_{0}$, the moving region $V(t)$ in the $\boldsymbol{r}$ - variables is replaced by the fixed region $V_{0}=V(0)$,

$$
\int_{V} f(\boldsymbol{r}, t) d^{3} r=\int_{V_{0}} f\left(\boldsymbol{r}_{0}, t\right) J d^{3} r_{0} .
$$

Hence,

$$
\begin{aligned}
\frac{d}{d t} \int_{V} f d^{3} r & =\int_{V_{0}}\left(J \frac{d f}{d t}+f \frac{d J}{d t}\right) d^{3} r_{0} \\
& =\int_{V_{0}}\left(\frac{d f}{d t}+f \nabla \cdot \boldsymbol{v}\right) J d^{3} r_{0} \\
& =\int_{V}\left(\frac{d f}{d t}+f \nabla \cdot \boldsymbol{v}\right) d^{3} r .
\end{aligned}
$$




$$
\frac{d f}{d t}=\frac{\partial f}{\partial t}+\boldsymbol{v} \cdot \nabla f
$$

Therefore,

$$
\frac{d}{d t} \int_{V} f d^{3} r=\int_{V}\left[\frac{\partial f}{\partial t}+\nabla \cdot(f \boldsymbol{v})\right] d^{3} r
$$

and

$$
\frac{d}{d t} \int_{V} f d^{3} r=\frac{\partial}{\partial t} \int_{V} f d^{3} r+\oint_{S} f \boldsymbol{v} \cdot d^{2} \boldsymbol{r},
$$

where $S$ is the bounding material surface. The differentiation in the first term of the right-hand side is taken with $V$ fixed so that, in particular,

$$
\frac{d V}{d t}=\oint_{S} \boldsymbol{v} \cdot d^{2} \boldsymbol{r}=\int_{V} \boldsymbol{\nabla} \cdot \boldsymbol{v} d^{3} r,
$$

gives the change in the volume of the piece of fluid as a result of the movement of its surface. In general, the transport theorem states that the rate of change of the integral of $f$ over a material volume $V$ equals the rate of change of $f$ integrated over the volume fixed in space that instantaneously coincides with $V$ plus the flux of $f$ out of the bounding surface.

\section{The equation of continuity}

The number of particles occupying a region $V$ is given by

$$
\int_{V} n(\boldsymbol{r}, t) d^{3} r
$$

The principle of conservation of the number of particles can be expressed by

$$
\frac{d}{d t} \int_{V} n(\boldsymbol{r}, t) d^{3} r=0
$$

and states that the number of particles in a material volume $V$ does not change as $V$ moves with the fluid. Using the transport theorem, this reduces to

$$
\int_{V}\left[\frac{\partial n}{\partial t}+\nabla \cdot(n \boldsymbol{v})\right] d^{3} r=0 .
$$

The volume $V$ being arbitrary, it follows that

$$
\frac{\partial n}{\partial t}+\nabla \cdot(n \boldsymbol{v})=0,
$$

which is the spatial (Eulerian) form of the equation of continuity. An alternative form is

$$
\frac{d n}{d t}+n \nabla \cdot v=0
$$

Multiplying this equation by $J$ and using Euler's formula for the Jacobian, the material (Lagrangian) form of the equation of continuity is obtained,

$$
\frac{d}{d t}(n J)=0 \Rightarrow n J=n_{0}
$$

with the initial density distribution $n_{0}=n_{0}\left(\boldsymbol{r}_{0}\right)$. Combining the transport theorem and the equation of continuity, one obtains

$$
\frac{d}{d t} \int_{V} n f d^{3} r=\int_{V} n \frac{d f}{d t} d^{3} r .
$$

\section{Transformation from Eulerian to Lagrangian coordinates}

Consider the transformation

$$
\boldsymbol{r}, t \rightarrow \boldsymbol{r}_{0}, \tau
$$

where $\boldsymbol{r}_{0}$ is the initial position of a fluid element (Lagrangian coordinate). The Eulerian variables $\boldsymbol{r}$ and $t$ are related to $\boldsymbol{r}_{0}$ and $\tau$ by

$$
\boldsymbol{r}=\boldsymbol{r}_{0}+\boldsymbol{\xi}\left(\boldsymbol{r}_{0}, \tau\right), \quad t=\tau,
$$

where $\xi$ is the Lagrangian displacement. It follows that

$$
\nabla_{0} \equiv\left(\nabla_{0} \boldsymbol{r}\right) \cdot \boldsymbol{\nabla}+\underbrace{\left(\nabla_{0} t\right)}_{0} \partial / \partial t \equiv\left(\overline{\bar{I}}+\nabla_{0} \boldsymbol{\xi}\right) \cdot \boldsymbol{\nabla} .
$$

Hence,

$$
\nabla \equiv\left(\overline{\bar{I}}+\nabla_{0} \xi\right)^{-1} \cdot \nabla_{0} \equiv \overline{\bar{J}}^{-1} \cdot \nabla_{0}
$$

and

$$
\nabla_{0} \equiv\left(\overline{\bar{I}}-\nabla_{0} \xi\right)^{-1} \cdot \nabla \equiv \overline{\bar{J}} \cdot \nabla
$$

Also,

$$
\frac{\partial}{\partial \tau} \equiv \frac{\partial \boldsymbol{r}}{\partial \tau} \cdot \boldsymbol{\nabla}+\frac{\partial t}{\partial \tau} \frac{\partial}{\partial t} \equiv \frac{\partial \boldsymbol{\xi}}{\partial \tau} \cdot \boldsymbol{\nabla}+\frac{\partial}{\partial t}
$$

and

$$
\frac{\partial}{\partial t} \equiv \frac{\partial}{\partial \tau}-\frac{\partial \xi}{\partial \tau} \cdot\left(\overline{\bar{I}}+\nabla_{0} \xi\right)^{-1} \cdot \nabla_{0}
$$

Using the definition of velocity, $\boldsymbol{v}=\partial \boldsymbol{\xi} / \partial \tau$,

$$
\boldsymbol{r}=\boldsymbol{r}_{0}+\boldsymbol{\xi}\left(\boldsymbol{r}_{0}, \tau\right)=\boldsymbol{r}_{0}+\int_{0}^{\tau} \boldsymbol{v}\left(\boldsymbol{r}_{0}, \tau^{\prime}\right) d \tau^{\prime} .
$$

The convective derivative, when expressed in Lagrangian coordinates, reduces to $\partial / \partial \tau$,

$$
\frac{\partial}{\partial t}+\boldsymbol{v} \cdot \boldsymbol{\nabla} \equiv \frac{\partial}{\partial \tau}
$$

\section{REFERENCES}

'J. W. Herivel, "The derivation of the equations of motion of an ideal fluid by Hamilton's principle," Math. Proc. Cambridge Philos. Soc. 51, 344-349 (1955).

${ }^{2}$ J. Serrin, "Mathematical principles of classical fluid mechanics," Handb. Phys. 3/8/1, 125-263 (1959).

${ }^{3}$ C. Eckart, "Variation principles of hydrodynamics," Phys. Fluids 3, 421-427 (1960).

${ }^{4}$ R. L. Seliger and G. B. Witham, "Variational principles in continuum mechanics," Proc. R. Soc. A 305, 1-25 (1968). 
${ }^{5}$ F. P. Bretherton, “A note on Hamilton's principle for perfect fluids," J. Fluid Mech. 44, 19-31 (1970).

${ }^{6} \mathrm{R}$. Jackiw, Lectures on Fluid Dynamics-A Particle Theorist's View of Supersymmetric, Non-Abelian, Noncommutative Fluid Mechanics and d-Branes (Springer-Verlag, New York, 2002).

${ }^{7}$ T. Kambe, "Variational formulation of ideal fluid flows according to gauge principle," Fluid Dyn. Res. 40, 399-426 (2008).

${ }^{8}$ G. Badin and F. Crisciani, Variational Formulation of Fluid and Geophysical Fluid Dynamics-Mechanics, Symmetries and Conservation Laws (Springer International Publishing AG, Cham, Switzerland, 2018).

${ }^{9}$ R. Salmon, "Hamiltonian fluid mechanics," Ann. Rev. Fluid Mech. 20, 225-256 (1988).

${ }^{10} \mathrm{~S}$. Weinberg, Gravitation and Cosmology: Principles and Applications of the General Theory of Relativit (John Wiley and Sons, New York, 1972).

${ }^{11}$ L. D. Landau and E. M. Lifshitz, Fluid Mechanics (Pergamon Press, Oxford, 1987).

${ }^{12}$ A. H. Taub, "General relativistic principle for perfect fluids," Phys. Rev. 94, 1468-1470 (1954).

${ }^{13}$ D. E. Soper, Classical Field Theory (John Wiley and Sons, New York, 1976).

${ }^{14} \mathrm{~L}$. Comisso and F. A. Asenjo, "Thermal-inertial effects on magnetic reconnection in relativistic pair plasmas," Phys. Rev. Lett. 113, 045001-045005 (2014).

${ }^{15}$ Y. Kawazura, G. Miloshevich, and P. J. Morrison, "Action principles for relativistic extended magnetohydrodynamics: A unified theory of magnetofluid models," Phys. Plasmas 24, 022103 (2017).

${ }^{16}$ A. L. Cauchy, "Théorie de la propagation des ondes à la surface d'un fluide pesant d'une profondeur indéfinie," Mém. Divers Savants 1, 5-318 (1815).

${ }^{17} \mathrm{H}$. K. Moffatt, "The degree of knottedness of tangled vortex lines," J. Fluid Mech. 35, 117-129 (1969).

${ }^{18} \mathrm{U}$. Frisch and B. Villone, "Cauchy's almost forgotten Lagrangian formulation of the Euler equation for 3D incompressible flow," arXiv:1402.4957v3 (2014).

${ }^{19}$ L. Woltjer, "A theorem on force-free magnetic fields," Proc. Natl. Acad. Sci. U. S. A. 44, 489-491 (1958).

${ }^{20}$ J. B. Taylor, "Relaxation of toroidal plasma and generation of reverse magnetic fields,” Phys. Rev. Lett. 33, 1139-1141 (1974).

${ }^{21}$ G. Webb, Magnetohydrodynamics and Fluid Dynamics: Action Principles and Conservation Laws (Springer International Publishing AG, Cham, Switzerland, 2018).

${ }^{22}$ I. B. Berstein, E. A. Frieman, M. D. Kruskal, and R. M. Kulsrud, "An energy principle for hydromagnetic stability problems,” Proc. R. Soc. London, Ser. A 244, 17-40 (1958).

${ }^{23}$ L. Woltjer, "On hydromagnetic equilibrium," Proc. Natl. Acad. Sci. U. S. A. 44, 833-841 (1958).

${ }^{24}$ G. O. Ludwig, "Relativistic fluid equations with applications to the study of instabilities in magnetized beam-plasma systems," Ph.D. thesis (Cornell University, Ithaca, NY, 1976).
${ }^{25}$ H. Goldstein, C. P. Poole, and J. L. Safko, Classical Mechanics, 3rd ed. (Addison Wesley, Boston, MA, 2001).

${ }^{26}$ G. F. Chew, M. L. Goldberger, and F. E. Low, "The Boltzmann equation and the one-fluid hydromagnetic equations in the absence of particle collisions," Proc. Roy. Soc. A A236, 112-118 (1956).

${ }^{27} \mathrm{G}$. O. Ludwig, "Relativistic distribution functions, fluid equations and equations of state for magnetized electron streams," Plasma Phys. Controlled Fusion 49, 1521-1549 (2007).

${ }^{28}$ M. J.-E. Manuel et al., "Magnetized disruption of inertially confined plasma flows," Phys. Rev. Lett. 122, 225001-225005 (2019).

${ }^{29}$ J. A. Stratton, Electromagnetic Theory (McGraw-Hill, New York, 1941).

${ }^{30}$ W. K. H. Panofsky and M. Phillips, Classical Electricity and Magnetism, 2nd ed. (Addison-Wesley, Reading, MA, 1962).

${ }^{31}$ J. D. Jackson, Classical Electrodynamics, 3rd ed. (John Wiley and Sons, Hoboken, NJ, 1998).

${ }^{32}$ L. D. Landau and E. M. Lifshitz, The Classical Theory of Fields, 4th ed. (Butterworth-Heinemann-Reed Elsevier, Oxford, 1996).

${ }^{33} \mathrm{H}$. Thirring, "Über die formale Analogie zwischen den elektromagnetischen Grundgleichugen und den Einsteischen Gravitationsgleichungen erster Näherung," Phys. Z. 19, 204-205 (1918).

${ }^{34}$ B. Mashoon, "Gravitoelectromagnetism: A brief review," arXiv:031103v2 [grqc]. (2008).

${ }^{35} \mathrm{H}$. Pfister, "Editorial note to: Hans Thirring, on the formal analogy between the basic electromagnetic equations and Einstein's gravity equations in first approximation," Gen. Relativ. Gravitation 44, 3217-3224 (2012).

${ }^{36}$ T. A. Moore, A General Relativity Workbook (University Science Books, Mill Valley, CA, 2013).

${ }^{37} \mathrm{~J}$. Lense and $\mathrm{H}$. Thirring, "Über den Einflu $\beta$ der Eigenrotation der Zentralkörper auf die Bewegung der Planeten und Monden nach der Einsteinschen Gravitationstheorie," Phys. Z. 19, 156-163 (1918).

${ }^{38}$ H. Pfister, "On the history of the so called Lense-Thirring effect," Gen. Relativ. Gravitation 39, 1735-1748 (2007).

${ }^{39}$ L. Iorio, H. I. M. Lichtenegger, M. L. Ruggiero, and C. Corda, "Phenomenology of the Lense-Thirring effect in the solar system," Astrophys. Space Sci. 331, 351-395 (2011).

${ }^{40}$ C. W. F. Everitt et al., "Gravity probe B: Final results of a space experiment to test general relativity,” Phys. Rev. Lett. 106(5), 221101 (2011).

${ }^{41}$ F. Jüttner, "Das Maxwellsche Gesetz der Geschwindigkeistverteilung in der relativtheorie," Ann. Phys. 339, 856-882 (1911).

${ }^{42}$ J. L. Synge, The Relativistic Gas (North-Holland, Amsterdam, 1957).

${ }^{43}$ S. R. de Groot, C. G. van Weert, W. T. Hermens, and W. A. van Leeuwen, "On relativistic gas theory. I. the second law for a gas mixture outside equilibrium," Physica 40, 257-276 (1968). 